TRANSACTIONS OF THE

AMERICAN MATHEMATICAL SOCIETY

Volume 365, Number 12, December 2013, Pages 6441-6469

S 0002-9947(2013)05863-1

Article electronically published on August 20, 2013

\title{
QUANTUM DIMENSIONS AND QUANTUM GALOIS THEORY
}

\author{
CHONGYING DONG, XIANGYU JIAO, AND FENG XU
}

\begin{abstract}
The quantum dimensions of modules for vertex operator algebras are defined and their properties are discussed. The possible values of the quantum dimensions are obtained for rational vertex operator algebras. A criterion for simple currents of a rational vertex operator algebra is given and a full Galois theory for rational vertex operator algebras is established using the quantum dimensions.
\end{abstract}

\section{INTRODUCTION}

The dimension of a space or an object is definitely an important concept in both mathematics and physics. The goal of the present paper is to give a systematic study of the "dimension" of a module over a vertex operator algebra. More precisely, we study how to define quantum dimensions and how to compute quantum dimensions and the possible values of quantum dimensions. The concept of quantum dimensions goes back to the physical literature [FMS], and most discussions on quantum dimensions focus on the rational vertex operator algebras associated to the affine Kac-Moody algebras [FZ, DL and Virasoro algebras DMZ, W]. The mathematical work on quantum dimensions has been limited to the conformal nets approach to conformal field theory [KLM where the quantum dimensions are called the statistical dimensions or the square root of index [J], $[\mathrm{PP}]$.

Our own motivation for studying quantum dimensions comes from trying to understand that the Galois theory for vertex operator algebras [DM1, DLM1, [HMT, originated from orbifold theory [FLM], DVVV], DLM5]. For a vertex operator algebra $V$ and a finite automorphism group $G$ of $V$, the fixed points $V^{G}$ are also a vertex operator algebra. It has already been established in [DM1] and [HMT that there is a one-to-one correspondence between the subgroups of $G$ and vertex operator subalgebras of $V$ containing $V^{G}$. To get a complete Galois theory for $V^{G} \subset V$, one needs a notion of "dimension" $\left[V: V^{G}\right]$ of $V$ over $V^{G}$ such that $\left[V: V^{G}\right]=o(G)$. Various efforts were tried without success until we turned our attention to the quantum dimensions. So as an application of the quantum dimensions we exhibit Galois theory for a vertex operator algebra $V$ together with a finite automorphism group $G$.

Let $V=\bigoplus_{n \in \mathbb{Z}} V_{n}$ be a vertex operator algebra and $M=\bigoplus_{n>0} M_{\lambda+n}$ a $V$ module. The quantum dimension qdim $\operatorname{dim}_{V} M$ of $M$ over $V$ is essentially the relative dimension $\frac{\operatorname{dim} M}{\operatorname{dim} V}$. Unfortunately, both $\operatorname{dim} M$ and $\operatorname{dim} V$ are infinite. One has to

Received by the editors January 12, 2012 and, in revised form, April 24, 2012.

2010 Mathematics Subject Classification. Primary 17B69.

The first author was supported by NSF grants and a faculty research fund from the University of California at Santa Cruz.

The third author was supported by an NSF grant and a faculty research fund from the University of California at Riverside. 
use limits to approach $\frac{\operatorname{dim} M}{\operatorname{dim} V}$. The original definition of $\operatorname{qdim}_{V} M$ involves the $q$ characters of $V$ and $M$. The $q$-character of $M$ is a formal power series

$$
\operatorname{ch}_{q} M=q^{\lambda-c / 24} \sum_{n=0}^{\infty}\left(\operatorname{dim} M_{\lambda+n}\right) q^{n},
$$

where $c$ is the central charge of $V$. It is proved in [Z] that the formal power series $\operatorname{ch}_{q} M$ converges to a holomorphic function in the upper half-plane in variable $\tau$ where $q=e^{2 \pi i \tau}$ under certain conditions. It is well known that $\operatorname{qdim}_{V} M$ can be defined as the limit of $\frac{\mathrm{ch}_{q} M}{\mathrm{ch}_{q} V}$ as $q$ goes to 1 from the left. The advantage of this definition is that one can use the modular transformation property of the $q$ characters $[\mathrm{Z}]$ and the Verlinde formula $[\mathrm{V},[\mathrm{H}]$ to compute the quantum dimensions and investigate their properties. In fact, we are following this approach closely in the present paper. The disadvantage of this definition is that it requires both rationality and $C_{2}$-cofiniteness of $V$. So this definition does not apply to irrational vertex operator algebras.

We propose two more definitions of quantum dimension, which work for any vertex operator algebra. The first one is given by the the limit of relative dimension $\lim _{n \rightarrow \infty} \frac{\operatorname{dim} M_{\lambda+n}}{\operatorname{dim} V_{n}}$ and the other is $\lim _{n \rightarrow \infty} \frac{\sum_{m=0}^{n} \operatorname{dim} M_{\lambda+m}}{\sum_{m=0}^{n} \operatorname{dim} V_{m}}$. It is proved that if $\lim _{n \rightarrow \infty} \frac{\operatorname{dim} M_{\lambda+n}}{\operatorname{dim} V_{n}}$ exists, then all the limits exist and are equal. The latter definition of quantum dimension tells us the real meaning of the quantum dimension. We firmly believe that these three definitions are equivalent, although we could not prove the assertion in this paper. We also suspect that for a simple vertex operator algebra, the quantum dimension of any irreducible module exists.

The quantum dimensions for rational and $C_{2}$-cofinite vertex operator algebras have two main properties: (a) The quantum dimension of a tensor product of two modules is the product of quantum dimensions; (b) An irreducible module is a simple current if and only if the quantum dimension is 1 . The first property which is true for the tensor product of vector spaces is important in many aspects. This implies that the quantum dimensions satisfy a certain system of equations of degree 2 with integral coefficients and helps to compute the quantum dimensions. The second property enables us to determine the simple currents when the quantum dimensions are easily calculated. For example, for a framed vertex operator algebra DGH, this can be easily done using the quantum dimensions for the rational vertex operator algebra $L\left(\frac{1}{2}, 0\right)$ associated to the Virasoro algebra with the central charge $\frac{1}{2}$.

It is worthy to mention that the minimal weight $\lambda_{\min }$ of the irreducible modules plays an essential role in computing the quantum dimensions using the $S$-matrix. For most rational vertex operator algebras including those associated to the unitary minimal series for the Virasoro algebra, $\lambda_{\min }=0$ is the weight of the vertex operator algebra $V$. The formula of the quantum dimensions in terms of the $S$ matrix is more or less standard. For an arbitrary vertex operator algebra such as those associated to the nonunitary minimal series for the Virasoro algebra, $\lambda_{\min }$ can be negative. We obtain a similar formula for the quantum dimensions using the irreducible module whose weight is $\lambda_{\min }$ instead of $V$. We also give two examples of nonunitary vertex operator algebras to illustrate this.

Finding the possible values of the quantum dimensions for a rational and $C_{2^{-}}$ cofinite vertex operator algebra is another task in this paper. With the help of 
the Perron-Frobenius Theorem on eigenvalues and some graph theory we show that the quantum dimension of an irreducible module can only take values in $\{2 \cos (\pi / n) \mid n \geq 3\} \cup[2, \infty)$ which are the square roots of the possible values of the index of subfactors of type II [J]. The connection between quantum dimensions and the index of subfactors is exciting but not surprising. There have been three approaches (algebraic, analytic and geometric) to two dimensional conformal field theory [BPZ, [MS] in mathematics. The basic tool in the algebraic approach is the vertex operator algebra and the analytic approach uses the conformal nets GL, Wa. Although the connection among different approaches has not been understood fully, constructing a conformal net from a rational vertex operator algebra and a rational vertex operator algebra from a conformal net is highly desirable. The connection between quantum dimension and index gives further evidence for the equivalence of algebraic and analytic approaches to two dimensional conformal field theory.

Motivated by the representation theories of both finite groups and finite dimensional associative algebras, the notion of global dimension for a vertex operator algebra is proposed using the quantum dimensions of irreducible modules. Although we have not done much in the present paper on global dimension, the application of global dimension in the classification of irreducible modules for orbifold and coset vertex operator algebras is visible. The main challenge is how to find an alternating definition without using the quantum dimensions of irreducible modules. One could classify the irreducible modules using the global dimension and the quantum dimensions of known irreducible modules. This will be very useful in studying the orbifold theory as in the case of conformal nets $[\mathrm{X}]$.

The paper is organized as follows. We give the basics, including the definition of twisted modules and important concepts such as rationality, regularity and $C_{2^{-}}$ cofiniteness [Z], DLM2 in Section 2. The quantum dimensions are defined in Section 3 with examples. Section 4 is devoted to the study of the properties of quantum dimensions. In particular, the connection between quantum dimension and modular invariance $[\mathrm{Z}$, the tensor product of modules $[\mathrm{H}$ and the Verlinde formula $[\mathrm{V}]$ is investigated in great depth. A characterization of simple currents in terms of quantum dimensions is given. We present results on possible values of the quantum dimensions in Section 5. In the last section we give a full Galois theory for a simple vertex operator algebra with a finite automorphism group.

We thank Terry Gannon for useful suggestions on the possible values of the quantum dimensions.

\section{Preliminary}

In this section, we recall the various notions of twisted modules for a vertex operator algebra following [DLM3]. We also define the terms of rationality, regularity, and $C_{2}$-cofiniteness from $[\mathrm{Z}$ and DLM2. In addition, we discuss the modular invariance property of the trace functions for a rational vertex operator algebra $[\mathbf{Z}]$.

2.1. Basics. A vertex operator algebra $V=\bigoplus_{n \in \mathbb{Z}} V_{n}$ (as defined in [FLM]) is said to be of CFT type if $V_{n}=0$ for negative $n$ and $V_{0}=\mathbb{C} 1$. 
Let $V$ be a vertex operator algebra and $g$ an automorphism of $V$ with finite order $T$. Decompose $V$ into eigenspaces of $g$ :

$$
V=\bigoplus_{r \in \mathbb{Z} / T \mathbb{Z}} V^{r}
$$

where $V^{r}=\left\{v \in V \mid g v=e^{-2 \pi i r / T} v\right\}$. We use $r$ to denote both an integer between 0 and $T-1$ and its residue class $\bmod T$ in this situation. Let $W\{z\}$ denote the space of a $W$-valued formal series in arbitrary complex powers of $z$ for a vector space $W$.

Definition 2.1. A weak $g$-twisted $V$-module $M$ is a vector space equipped with a linear map

$$
\begin{aligned}
Y_{M}: V & \rightarrow(\text { End } M)\{z\}, \\
v & \mapsto Y_{M}(v, z)=\sum_{n \in \mathbb{Q}} v_{n} z^{-n-1} \quad\left(v_{n} \in \text { End } M\right),
\end{aligned}
$$

which satisfies the following: for all $0 \leq r \leq T-1, u \in V^{r}, v \in V, w \in M$,

$$
\begin{gathered}
Y_{M}(u, z)=\sum_{n \in \frac{r}{T}+\mathbb{Z}} u_{n} z^{-n-1}, \\
u_{l} w=0 \text { for } l \gg 0, \\
Y_{M}(\mathbf{1}, z)=I d_{M}, \\
z_{0}^{-1} \delta\left(\frac{z_{1}-z_{2}}{z_{0}}\right) Y_{M}\left(u, z_{1}\right) Y_{M}\left(v, z_{2}\right)-z_{0}^{-1} \delta\left(\frac{z_{2}-z_{1}}{-z_{0}}\right) Y_{M}\left(v, z_{2}\right) Y_{M}\left(u, z_{1}\right) \\
=z_{2}^{-1}\left(\frac{z_{1}-z_{0}}{z_{2}}\right)^{-r / T} \delta\left(\frac{z_{1}-z_{0}}{z_{2}}\right) Y_{M}\left(Y\left(u, z_{0}\right) v, z_{2}\right),
\end{gathered}
$$

where $\delta(z)=\sum_{n \in \mathbb{Z}} z^{n}$ (elementary properties of the $\delta$-function can be found in [FLM] ) and all binomial expressions (here and below) are to be expanded in nonnegative integral powers of the second variable.

Definition 2.2. A $g$-twisted $V$-module is a weak $g$-twisted $V$-module $M$ which carries a $\mathbb{C}$-grading induced by the spectrum of $L(0)$ where $L(0)$ is the component operator of $Y(\omega, z)=\sum_{n \in \mathbb{Z}} L(n) z^{-n-2}$. That is, we have

$$
M=\bigoplus_{\lambda \in \mathbb{C}} M_{\lambda},
$$

where $M_{\lambda}=\{w \in M \mid L(0) w=\lambda w\}$. Moreover, we require that $\operatorname{dim} M_{\lambda}$ is finite, and for fixed $\lambda, M_{\frac{n}{T}+\lambda}=0$ for all small enough integers $n$.

In this situation, if $w \in M_{\lambda}$ we refer to $\lambda$ as the weight of $w$ and write $\lambda=\operatorname{wt} w$. The totality of $g$-twisted $V$-modules defines a full subcategory of the category of $g$-twisted weak $V$-modules.

Denote the set of nonnegative integers by $\mathbb{Z}_{+}$. An important and related class of $g$-twisted modules is the following.

Definition 2.3. An admissible $g$-twisted $V$-module is a weak $g$-twisted $V$-module $M$ that carries a $\frac{1}{T} \mathbb{Z}_{+}$-grading

$$
M=\bigoplus_{n \in \frac{1}{T} \mathbb{Z}_{+}} M(n),
$$


which satisfies the following:

$$
v_{m} M(n) \subseteq M(n+\mathrm{wt} v-m-1)
$$

for homogeneous $v \in V, m \in \frac{1}{T} \mathbb{Z}$.

If $g=I d_{V}$ we have the notions of weak, ordinary and admissible $V$-modules DLM2.

If $M=\bigoplus_{n \in \frac{1}{T} \mathbb{Z}_{+}} M(n)$ is an admissible $g$-twisted $V$-module, the contragredient module $M^{\prime}$ is defined as follows:

$$
M^{\prime}=\bigoplus_{n \in \frac{1}{T} \mathbb{Z}_{+}} M(n)^{*},
$$

where $M(n)^{*}=\operatorname{Hom}_{\mathbb{C}}(M(n), \mathbb{C})$. The vertex operator $Y_{M^{\prime}}(a, z)$ is defined for $a \in V$ via

$$
\left\langle Y_{M^{\prime}}(a, z) f, u\right\rangle=\left\langle f, Y_{M}\left(e^{z L(1)}\left(-z^{-2}\right)^{L(0)} a, z^{-1}\right) u\right\rangle,
$$

where $\langle f, w\rangle=f(w)$ is the natural paring $M^{\prime} \times M \rightarrow \mathbb{C}$. One can prove (cf. [FHL], $[\mathrm{Xu}]$ ) the following:

Lemma 2.4. $\left(M^{\prime}, Y_{M^{\prime}}\right)$ is an admissible $g^{-1}$-twisted $V$-module.

We can also define the contragredient module $M^{\prime}$ for a $g$-twisted $V$-module $M$. In this case, $M^{\prime}$ is a $g^{-1}$-twisted $V$-module. Moreover, $M$ is irreducible if and only if $M^{\prime}$ is irreducible.

Definition 2.5. A vertex operator algebra $V$ is called $g$-rational, if the admissible $g$-twisted module category is semisimple. $V$ is called rational if $V$ is 1-rational.

The following lemma about $g$-rational vertex operator algebras is well known DLM3.

Lemma 2.6. If $V$ is g-rational and $M$ is an irreducible admissible g-twisted $V$ module, then

(1) $M$ is a g-twisted $V$-module and there exists a number $\lambda \in \mathbb{C}$ such that $M=\bigoplus_{n \in \frac{1}{T} \mathbb{Z}_{+}} M_{\lambda+n}$ where $M_{\lambda} \neq 0$. The $\lambda$ is called the conformal weight of $M$.

(2) There are only finitely many irreducible admissible g-twisted $V$-modules up to isomorphism.

Besides rationality, there is another important concept called $C_{2}$-cofiniteness $[\mathrm{Z}]$.

Definition 2.7. We say that a VOA $V$ is $C_{2}$-cofinite if $V / C_{2}(V)$ is finite dimensional, where $C_{2}(V)=\left\langle v_{-2} u \mid v, u \in V\right\rangle$.

For a simple vertex operator algebra $V$ which has finitely many irreducible modules, we always denote by $M^{0}, M^{1}, \cdots, M^{d}$ all the inequivalent irreducible $V$ modules with $M^{0} \cong V$. Also, let $\lambda_{i}$ denote the conformal weight of $M^{i}$. The following theorem is proved in DLM5].

Theorem 2.8. Let $V$ be a rational and $C_{2}$-cofinite vertex operator algebra. Then $\lambda_{i}$ are rationals for all $i$.

Definition 2.9. A vertex operator algebra $V$ is called regular if every weak $V$ module is a direct sum of irreducible $V$-modules. 
Remark 2.10. It is proved in $\mathrm{ABD}$ that if $V$ is of CFT type, then regularity is equivalent to rationality and $C_{2}$-cofiniteness. Also, $V$ is regular if and only if the weak module category is semisimple [DY].

Let $V$ be a vertex operator algebra. In DLM4 a series of associative algebras $A_{n}(V)$ was introduced for nonnegative integers $n$. In the case $n=0, A_{0}(V)=A(V)$ is Zhu's algebra as defined in [Z]. We briefly review $A_{n}(V)$ here. For homogeneous $u, v \in V$ we define

$$
\begin{aligned}
& u \circ_{n} v=\operatorname{Res}_{z} Y(u, z) v \frac{(1+z)^{w t u+n}}{z^{2 n+2}}, \\
& u *_{n} v=\sum_{m=0}^{n}(-1)^{m}\left(\begin{array}{c}
m+n \\
n
\end{array}\right) \operatorname{Res}_{z} Y(u, z) \frac{(1+z)^{w t u+n}}{z^{n+m+1}} .
\end{aligned}
$$

Extend $\circ_{n}$ and $*_{n}$ linearly to obtain bilinear products on $V$. We let $O_{n}(V)$ be the linear span of all $u \circ_{n} v$ and $L(-1) u+L(0) u$. We have (see [DLM4, [Z]):

Theorem 2.11. Let $V$ be a vertex operator algebra and $M=\bigoplus_{n=0}^{\infty} M(n)$ be an admissible $V$-module. Set $A_{n}(V)=V / O_{n}(V)$. Then

(1) For any $n \in \mathbb{Z}_{+}, A_{n}(V)$ is an associative algebra with respect to $*_{n}$.

(2) For $0 \leq m \leq n, M(m)$ is an $A_{n}(V)$-module.

(3) If $V$ is rational, then for any $n \in \mathbb{Z}_{+}, A_{n}(V)$ is a finite dimensional semisimple associative algebra.

(4) If $V$ is a simple rational vertex operator algebra and $\left\{M^{i} \mid i=0, \cdots, d\right\}$ are the inequivalent irreducible $V$-modules, then $A_{n}(V) \cong \bigoplus_{i=0}^{d} \bigoplus_{m \leq n} \operatorname{End} M^{i}(m)$.

2.2. Modular invariance of trace functions. We now turn our discussion to the modular-invariance property in VOA theory. The most basic function is the formal character of a $g$-twisted $V$-module $M=\bigoplus_{n \in \frac{1}{T} \mathbb{Z}_{+}} M_{\lambda+n}$. We define the formal character of $M$ as

$$
\operatorname{ch}_{q} M=\operatorname{tr}_{M} q^{L(0)-c / 24}=q^{\lambda-c / 24} \sum_{n \in \frac{1}{T} \mathbb{Z}_{+}}\left(\operatorname{dim} M_{\lambda+n}\right) q^{n},
$$

where $\lambda$ is the conformal weight of $M$. It is proved in [Z] and DLM5] that $\operatorname{ch}_{q} M$ converges to a holomorphic function on the domain $|q|<1$ if $V$ is $C_{2}$-cofinite. We sometimes also use $Z_{M}(\tau)$ to denote the holomorphic function $\operatorname{ch}_{q} M$. Here and below $\tau$ is in the complex upper half-plane $\mathbf{H}$ and $q=e^{2 \pi i \tau}$.

For any homogeneous element $v \in V$ we define a trace function associated to $v$ as follows:

$$
Z_{M}(v, \tau)=\operatorname{tr}_{M} o(v) q^{L(0)-c / 24}=q^{\lambda-c / 24} \sum_{n \in \frac{1}{T} \mathbb{Z}_{+}} \operatorname{tr}_{M_{\lambda+n}} o(v) q^{n},
$$

where $o(v)=v($ wt $v-1)$ is the degree zero operator of $v$.

There is a natural action of Aut $(V)$ on twisted modules [DLM5]. Let $g, h$ be two automorphisms of $V$ with $g$ of finite order. If $\left(M, Y_{g}\right)$ is a weak $g$-twisted $V$-module, there is a weak $h g h^{-1}$-twisted $V$-module $\left(M \circ h, Y_{h g h^{-1}}\right)$ where $M \circ h \cong M$ as vector spaces and

$$
Y_{h g h^{-1}}(v, z)=Y_{g}\left(h^{-1} v, z\right)
$$

for $v \in V$. This defines a left action of $\operatorname{Aut}(V)$ on weak twisted $V$-modules and on isomorphism classes of weak twisted $V$-modules. Symbolically, we write

$$
h \circ\left(M, Y_{g}\right)=\left(M \circ h, Y_{h g h^{-1}}\right)=h \circ M,
$$


where we sometimes abuse notation slightly by identifying $\left(M, Y_{g}\right)$ with the isomorphism class that it defines.

If $g, h$ commute, obviously $h$ acts on the $g$-twisted modules as above. We set $\mathscr{M}(g)$ to be the equivalence classes of irreducible $g$-twisted $V$-modules and $\mathscr{M}(g, h)=\{M \in \mathscr{M}(g) \mid h \circ M \cong M\}$. Then for any $M \in \mathscr{M}(g, h)$, there is a $g$-twisted $V$-module isomorphism

$$
\varphi(h): h \circ M \rightarrow M .
$$

The linear map $\varphi(h)$ is unique up to a nonzero scalar. We set

$$
Z_{M}(g, h, \tau)=\operatorname{tr}_{M} \varphi(h) q^{L(0)-c / 24}=q^{\lambda-c / 24} \sum_{n \in \frac{1}{T} \mathbb{Z}_{+}} \operatorname{tr}_{M_{\lambda+n}} \varphi(h) q^{n} .
$$

Since $\varphi(h)$ is unique up to a nonzero scalar, $Z_{M}(g, h, \tau)$ is also defined up to a nonzero scalar. The choice of the scalar does not interfere with any of the results in this paper. For a homogeneous element $v \in V$ and any commuting pair $(g, h)$ we define

$$
T_{M}(v, g, h, \tau)=q^{\lambda-c / 24} \sum_{n \in \frac{1}{T} \mathbb{Z}_{+}} \operatorname{tr}_{M_{\lambda+n}} o(v) \varphi(h) q^{n},
$$

where $M \in \mathscr{M}(g, h)$. It is easy to see when $v=\mathbf{1}, T_{M}(1, g, h, \tau)=Z_{M}(g, h, \tau)$.

Zhu has introduced a second vertex operator algebra $(V, Y[], \mathbf{1}, \tilde{\omega})$ associated to $V$ in $[$ Z]. Here $\tilde{\omega}=\omega-c / 24$ and

$$
Y[v, z]=Y\left(v, e^{z}-1\right) e^{z \cdot \mathrm{wt} v}=\sum_{n \in \mathbb{Z}} v[n] z^{n-1}
$$

for homogeneous $v$. We also write

$$
Y[\tilde{\omega}, z]=\sum_{n \in \mathbb{Z}} L[n] z^{-n-2} .
$$

We must take care to distinguish between the notion of conformal weight in the original vertex operator algebra and in the second vertex operator algebra $(Y, Y[], \mathbf{1}, \tilde{\omega})$. If $v \in V$ is homogeneous in the second vertex operator algebra, we denote its weight by wt $[v]$. For such $v$ we define an action of the modular group $\Gamma$ on $T_{M}$ in a familiar way, namely

$$
\left.T_{M}\right|_{\gamma}(v, g, h, \tau)=(c \tau+d)^{-\mathrm{wt}[v]} T_{M}(v, g, h, \gamma \tau),
$$

where $\gamma \tau$ is the Möbius transformation; that is,

$$
\gamma: \tau \mapsto \frac{a \tau+b}{c \tau+d}, \quad \gamma=\left(\begin{array}{ll}
a & b \\
c & d
\end{array}\right) \in \Gamma=S L(2, \mathbb{Z}) .
$$

Let $P(G)$ denote the commuting pairs of elements in a group $G$. We let $\gamma \in \Gamma$ act on the right of $P(G)$ via

$$
(g, h) \gamma=\left(g^{a} h^{c}, g^{b} h^{d}\right) .
$$

The following theorem is proved in [Z], DLM5].

Theorem 2.12. Assume $(g, h) \in P(\operatorname{Aut}(V))$ such that the orders of $g$ and $h$ are finite. Let $\gamma=\left(\begin{array}{ll}a & b \\ c & d\end{array}\right) \in \Gamma$. Also assume that $V$ is $g^{a} h^{c}$-rational and $C_{2}$-cofinite. 
If $M^{i}$ is an irreducible $h$-stable $g$-twisted $V$-module, then

$$
\left.T_{M^{i}}\right|_{\gamma}(v, g, h, \tau)=\sum_{N_{j} \in \mathscr{M}\left(g^{a} h^{c}, g^{b} h^{d}\right)} \gamma_{i, j}(g, h) T_{N_{j}}(v,(g, h) \gamma, \tau),
$$

where $\gamma_{i, j}(g, h)$ are some complex numbers independent of the choice of $v \in V$.

Remark 2.13. In the case $g=h=1$ and $\gamma=S=\left(\begin{array}{cc}0 & -1 \\ 1 & 0\end{array}\right)$ we have:

$$
Z_{M^{i}}\left(v,-\frac{1}{\tau}\right)=\tau^{\mathrm{wt}[v]} \sum_{j=0}^{d} S_{i, j} Z_{M^{j}}(v, \tau) .
$$

The matrix $S=\left(S_{i, j}\right)$ is called an $S$-matrix which is independent of the choice of $v$.

\section{QuANTUM DIMENSION}

In this section we define the quantum dimension for an ordinary $V$-module $M$ and give some examples.

\subsection{Definition.}

Definition 3.1. Let $V$ be a vertex operator algebra and $M$ a $V$-module such that $Z_{V}(\tau)$ and $Z_{M}(\tau)$ exist. The quantum dimension of $M$ over $V$ is defined as

$$
\operatorname{qdim}_{V} M=\lim _{y \rightarrow 0} \frac{Z_{M}(i y)}{Z_{V}(i y)}
$$

where $y$ is real and positive.

Remark 3.2. Sometimes we use an alternative definition which involves the $q$ characters:

$$
\operatorname{qdim}_{V} M=\lim _{q \rightarrow 1^{-}} \frac{\operatorname{ch}_{q} M}{\operatorname{ch}_{q} V}
$$

This is because we know $\tau=i y \rightarrow 0, q=e^{2 \pi i \tau}=e^{-2 \pi y} \rightarrow 1^{-}$. This definition of quantum dimension seems well known in the literature.

Remark 3.3. Quantum dimension is formally defined. Intuitively, for an arbitrary vertex operator algebra $V$ and any $V$-module $M, q \operatorname{dim}_{V} M$ might not exist. But we will prove that for rational and $C_{2}$-cofinite vertex operator algebras, quantum dimensions do exist.

Remark 3.4. If qdim $V$ exists, then it is nonnegative.

Remark 3.5. If $\operatorname{qdim}_{V} M$ exists, then

$$
\operatorname{qdim}_{V} M=\operatorname{qdim}_{V} M^{\prime} .
$$

In the definition of quantum dimensions one needs the convergence of both $\operatorname{ch}_{q} V$ and $\operatorname{ch}_{q} M$. But there is no theorem which guarantees the convergence of these 
formal $q$-characters for an arbitrary vertex operator algebra. It is natural to seek other equivalent definitions of quantum dimensions without using the convergence of formal characters.

Proposition 3.6. Let $V=\bigoplus_{n=0}^{\infty} V_{n}$ be a vertex operator algebra, and let $M=$ $\bigoplus_{n=0}^{\infty} M(n)$ be an admissible $V$-module with $\operatorname{dim} M(n)<\infty, \forall n \geq 0$. If $\lim _{n \rightarrow \infty} \frac{\operatorname{dim} M(n)}{\operatorname{dim} V_{n}}=d$ (finite or infinite) exists, then
(1) $\lim _{n \rightarrow \infty} \frac{\sum_{i=0}^{n} \operatorname{dim} M(i)}{\sum_{i=0}^{n} \operatorname{dim} V_{i}}=d$,
(2) $\lim _{q \rightarrow 1^{-}} \frac{\mathrm{ch}_{q} M}{\operatorname{ch}_{q} V}=d$.

Proof. For simplicity, we denote $\operatorname{dim} M(n)$ by $a_{n}$ and $\operatorname{dim} V_{n}$ by $b_{n}$. We first deal with the case that $d \neq \infty$. Then $\lim _{n \rightarrow \infty} \frac{a_{n}}{b_{n}}=d$ implies for any $\varepsilon>0,\left|\frac{a_{n}}{b_{n}}-d\right|<\varepsilon$, for $n \geq N$, where $N$ is a sufficiently large integer. Thus we have

$$
\left|a_{n}-b_{n} d\right|<\varepsilon b_{n} .
$$

For (1) we consider the following estimation:

$$
\begin{aligned}
\left|\frac{\sum_{n=0}^{m} a_{n}}{\sum_{n=0}^{m} b_{n}}-d\right| & =\left|\frac{\sum_{n=0}^{m} a_{n}-\sum_{n=0}^{m} b_{n} d}{\sum_{n=0}^{m} b_{n}}\right| \\
& =\left|\frac{\sum_{n=0}^{m}\left(a_{n}-b_{n} d\right)}{\sum_{n=0}^{m} b_{n}}\right| \\
& \leq\left|\frac{\sum_{n=0}^{N}\left(a_{n}-b_{n} d\right)}{\sum_{n=0}^{m} b_{n}}\right|+\left|\frac{\sum_{n=N+1}^{m}\left(a_{n}-b_{n} d\right)}{\sum_{n=0}^{m} b_{n}}\right| \\
& \leq\left|\frac{\sum_{n=0}^{N}\left(a_{n}-b_{n} d\right)}{\sum_{n=0}^{m} b_{n}}\right|+\left|\frac{\sum_{n=N+1}^{m} \varepsilon b_{n}}{\sum_{n=0}^{m} b_{n}}\right| \\
& \leq\left|\frac{\sum_{n=0}^{N}\left(a_{n}-b_{n} d\right)}{\sum_{n=0}^{m} b_{n}}\right|+\varepsilon .
\end{aligned}
$$

For the first term above, we know that $\lim _{m \rightarrow \infty} \sum_{n=0}^{m} b_{n}=\infty$, and the top term $\sum_{n=0}^{N}\left(a_{n}-b_{n} d\right)$ is a fixed number. So we can take $m>N_{1}$, for some big enough number $N_{1}$, and we get

$$
\left|\frac{\sum_{n=0}^{N}\left(a_{n}-b_{n} d\right)}{\sum_{n=0}^{m} b_{n}}\right|<\varepsilon .
$$

So for $m>\max \left\{N, N_{1}\right\},\left|\frac{\sum_{n=0}^{m} a_{n}}{\sum_{n=0}^{m} b_{n}}-d\right|<2 \varepsilon$, i.e.

$$
\lim _{n \rightarrow \infty} \frac{\sum_{i=0}^{n} \operatorname{dim} M(i)}{\sum_{i=0}^{n} \operatorname{dim} V_{i}}=d .
$$


(2) is proved in a similar way:

$$
\begin{aligned}
\left|\frac{\sum_{n=0}^{\infty} a_{n} q^{n}}{\sum_{n=0}^{\infty} b_{n} q^{n}}-d\right| & =\left|\frac{\sum_{n=0}^{\infty}\left(a_{n}-b_{n} d\right) q^{n}}{\sum_{n=0}^{\infty} b_{n} q^{n}}\right| \\
& =\left|\frac{\sum_{n=0}^{N}\left(a_{n}-b_{n} d\right) q^{n}}{\sum_{n=0}^{\infty} b_{n} q^{n}}+\frac{\sum_{n=N+1}^{\infty}\left(a_{n}-b_{n} d\right) q^{n}}{\sum_{n=0}^{\infty} b_{n} q^{n}}\right| \\
& \leq\left|\frac{\sum_{n=0}^{N}\left(a_{n}-b_{n} d\right) q^{n}}{\sum_{n=0}^{\infty} b_{n} q^{n}}\right|+\sum_{n=N+1}^{\infty}\left|\frac{\left(a_{n}-b_{n} d\right) q^{n}}{\sum_{n=0}^{\infty} b_{n} q^{n}}\right| \\
& \leq\left|\frac{\sum_{n=0}^{N}\left(a_{n}-b_{n} d\right) q^{n}}{\sum_{n=0}^{\infty} b_{n} q^{n}}\right|+\sum_{n=N+1}^{\infty}\left|\frac{\left(\varepsilon b_{n}\right) q^{n}}{\sum_{n=0}^{\infty} b_{n} q^{n}}\right| \\
& \leq\left|\frac{\sum_{n=0}^{N}\left(a_{n}-b_{n} d\right) q^{n}}{\sum_{n=0}^{\infty} b_{n} q^{n}}\right|+\varepsilon .
\end{aligned}
$$

Obviously, $\lim _{q \rightarrow 1^{-}}\left|\frac{\sum_{n=0}^{N}\left(a_{n}-b_{n} d\right) q^{n}}{\sum_{n=0}^{\infty} b_{n} q^{n}}\right|=0$. Thus when $q$ is close to 1 , the first term above is very small, and so is $\left|\frac{\sum_{n=0}^{\infty} a_{n} q^{n}}{\sum_{n=0}^{\infty} b_{n} q^{n}}-d\right|$. This shows that $\lim _{q \rightarrow 1^{-}} \frac{\operatorname{ch}_{q} M}{\operatorname{ch}_{q} V}=d$.

If $d=\infty$, it is evident that $\lim _{n \rightarrow \infty} \frac{\sum_{i=0}^{n} \operatorname{dim} M(i)}{\sum_{i=0}^{n} \operatorname{dim} V_{i}}=\infty$ and $\lim _{q \rightarrow 1^{-}} \frac{\mathrm{ch}_{q} M}{\mathrm{ch}_{q} V}=\infty$. The proof is complete.

Remark 3.7. We believe that for a simple vertex operator algebra $V$ and an irreducible $V$-module $M$, if one of the limits $\lim _{n \rightarrow \infty} \frac{\operatorname{dim} M(n)}{\operatorname{dim} V_{n}}, \lim _{n \rightarrow \infty} \frac{\sum_{i=0}^{n} \operatorname{dim} M(i)}{\sum_{i=0}^{n} \operatorname{dim} V_{i}}$ and $\lim _{q \rightarrow 1^{-}} \frac{\operatorname{ch}_{q} M}{\operatorname{ch}_{q} V}$ exists and is finite, then the other limits also exist and all limits are equal. But we cannot establish this result in the paper. There are counterexamples in pure analysis: If $a_{n}, b_{n}$ are nonnegative real numbers for $n \geq 0$ such that $\lim _{n \rightarrow \infty} \frac{\sum_{m=0}^{n} b_{m}}{\sum_{m=0}^{n} a_{m}}$ exists and is finite, then $\lim _{n \rightarrow \infty} \frac{b_{n}}{a_{n}}=\infty$. So the equality of these three limits has some deep reason (which we do not know) from the theory of vertex operator algebra.

If $V$ is a vertex operator algebra with only finitely many inequivalent irreducible modules, say $M^{0}, \cdots, M^{d}$, we give the definition of global dimension.

Definition 3.8. The global dimension of $V$ is defined as

$$
\operatorname{glob}(V)=\sum_{i=0}^{d}\left(\operatorname{qdim}_{V} M^{i}\right)^{2} .
$$

Remark 3.9. In the index theory of conformal nets, the so-called $\mu$-index which is similar to the global dimension defined above plays an important role in orbifold theory. We expect that we can use global dimensions to get some significant results in vertex operator algebra theory. The same global dimension is also defined in the setting of fusion category [ENO].

Here is a result on the global dimension for a rational vertex operator algebra using $A_{n}(V)$.

Proposition 3.10. Let $V$ be a rational vertex operator algebra and

$$
M^{i}=\bigoplus_{n \geq 0} M^{i}(n)
$$


be the irreducible modules with $M^{i}(0) \neq 0$ for all $i$. We assume $\lim _{n \rightarrow \infty} \frac{\operatorname{dim} M^{i}(n)}{\operatorname{dim} V_{i}}$ exists and is finite for all $i$. Then

$$
\operatorname{glob}(V)=\lim _{n \rightarrow \infty} \frac{\operatorname{dim} A_{n}(V) / A_{n-1}(V)}{\left(\operatorname{dim} V_{n}\right)^{2}} .
$$

Proof. It follows from DLM4] that $A_{n}(V) \cong \bigoplus_{i=0}^{d} \bigoplus_{m \leq n} \operatorname{End} M^{i}(m)$. Then

$$
A_{n}(V) / A_{n-1}(V) \cong \bigoplus_{i=0}^{d} \operatorname{End} M^{i}(n) .
$$

Using Proposition 3.6 and the definition of global dimension gives the result immediately.

3.2. Examples. We use the definition to compute the quantum dimensions of modules for Heisenberg vertex operator algebras and Virasoro vertex operator algebras.

Example 3.11. Let $M(1)$ be the Heisenberg vertex operator algebra constructed from the vector space $H$ of dimension $d$ and with a nondegenerate symmetric bilinear form. It is well known that every irreducible $M(1)$-module is of the form $M(1, \lambda), \lambda \in H$ with $q$-character

$$
\operatorname{ch}_{q} M(1, \lambda)=\frac{q^{\frac{(\lambda, \lambda)}{2}-\frac{d}{24}}}{\prod_{n \geq 1}\left(1-q^{n}\right)^{d}} .
$$

Using the alternating definition in Remark 3.2 we have

$$
\operatorname{qdim}_{M(1)} M(1, \lambda)=\lim _{q \rightarrow 1^{-}} \frac{\frac{q^{\frac{(\lambda, \lambda)}{2}}}{\prod_{n \geq 1}\left(1-q^{n}\right)^{d}}}{\frac{1}{\prod_{n \geq 1}\left(1-q^{n}\right)^{d}}}=1 .
$$

Example 3.12. Let $c$ and $h$ be two complex numbers and let $L(c, h)$ be the lowest weight irreducible module for the Virasoro algebra with central charge $c$ and lowest weight $h$. Then $L(c, 0)$ has a natural vertex operator algebra structure [FZ]. We are interested in the vertex operator algebra $L(1,0)$ and its irreducible modules. We know from $[\mathrm{KR}$ that

$$
\operatorname{dim}_{q} L(1, h)= \begin{cases}\frac{q^{\frac{n^{2}}{4}}-q^{\frac{(n+2)^{2}}{4}}}{\eta(q)} & \text { if } h=\frac{n^{2}}{4}, n \in \mathbb{Z} \\ \frac{q^{h}}{\eta(q)} & \text { otherwise }\end{cases}
$$

where

$$
\eta(q)=q^{1 / 24} \prod_{n=1}^{\infty}\left(1-q^{n}\right) .
$$

Then if $h \neq \frac{n^{2}}{4}$ for any integer $n$,

$$
\operatorname{qdim}_{L(1,0)} L(1, h)=\lim _{q \rightarrow 1^{-}} \frac{\frac{q^{h}}{\prod_{n \geq 1}\left(1-q^{n}\right)}}{\frac{1}{\prod_{n>1}\left(1-q^{n}\right)}}=\lim _{q \rightarrow 1^{-}} \frac{q^{h}}{1-q}=\infty .
$$


Similarly for $h=\frac{m^{2}}{4}$ for some integer $m$,

$$
\begin{aligned}
\operatorname{qdim}_{L(1,0)} L\left(1, \frac{m^{2}}{4}\right) & =\lim _{q \rightarrow 1^{-}} \frac{q^{\frac{m^{2}}{4}}-q^{\frac{(m+2)^{2}}{4}}}{1-q} \\
& =\lim _{q \rightarrow 1^{-}} \frac{q^{\frac{m^{2}}{4}}\left(1-q^{m+1}\right)}{1-q} \\
& =\lim _{q \rightarrow 1^{-}} q^{\frac{m^{2}}{4}}\left(1+q+\cdots+q^{m}\right) \\
& =m+1 .
\end{aligned}
$$

\section{Quantum dimensions And the Verlinde formula}

Computing quantum dimensions directly by using the definition is not easy. However, the definition of quantum dimensions involves the $q$-characters. This motivates us to use the modular invariant property of the trace functions $[\mathrm{Z}]$ to do the computation. It turns out that the Verlinde formula $[\mathrm{V}],[\mathrm{H}]$ plays an important role.

4.1. Verlinde formula. The Verlinde conjecture [V] in conformal field theory states that the action of the modular transformation $\tau \rightarrow-1 / \tau$ on the space of characters of a rational conformal field theory diagonalizes the fusion rules. In this section, we quote some results from $[\mathrm{H}]$ about the Verlinde conjecture of rational vertex operator algebras.

Let $V$ be a rational and $C_{2}$-cofinite simple VOA, and let $M^{0}, M^{1}, \cdots, M^{d}$ be as before. We use $N_{i, j}^{k}$ to denote $\operatorname{dim} I_{V}\left(\begin{array}{c}M^{k} \\ M^{i} M^{j}\end{array}\right)$, where $I_{V}\left(\begin{array}{c}M^{k} \\ M^{i} M^{j}\end{array}\right)$ is the space of intertwining operators of type $\left(\begin{array}{c}M^{k} \\ M^{i} M^{j}\end{array}\right) \cdot N_{i, j}^{k}$ are called the fusion rules. As usual, we use $M^{i^{\prime}}$ to denote $\left(M^{i}\right)^{\prime}$, the contragredient module of $M^{i}$. $[\mathrm{H}$.

The following theorem which plays an essential role in this section is proved in

Theorem 4.1. Let $V$ be a rational and $C_{2}$-cofinite simple vertex operator algebra of CFT type and assume $V \cong V^{\prime}$. Let $S=\left(S_{i, j}\right)_{i, j=0}^{d}$ be the $S$-matrix as defined in (2.2). Then

(1) $\left(S^{-1}\right)_{i, j}=S_{i, j^{\prime}}=S_{i^{\prime}, j}$, and $S_{i^{\prime}, j^{\prime}}=S_{i, j}$.

(2) $S$ is symmetric and $S^{2}=\left(\delta_{i, j^{\prime}}\right)$.

(3) $N_{i, j}^{k}=\sum_{s=0}^{d} \frac{S_{j, s} S_{i, s} S_{s, k}^{-1}}{S_{0, s}}$.

(4) The $S$-matrix diagonalizes the fusion matrix $N(i)=\left(N_{i, j}^{k}\right)_{j, k=0}^{d}$ with diagonal entries $\frac{S_{i, s}}{S_{0, s}}$, for $i, s=0, \cdots, d$. More explicitly, $S^{-1} N(i) S=\operatorname{diag}\left(\frac{S_{i, s}}{S_{0, s}}\right)_{s=0}^{d}$. In particular, $S_{0, s} \neq 0$ for $s=0, \cdots, d$.

4.2. Properties of quantum dimensions. We prove that the quantum dimensions of modules exist and are related to the $S$-matrix under certain assumptions in this subsection. Using the Verlinde formula we obtain an expression of the global dimension. We also show that quantum dimensions are multiplicative under tensor product, and give a criterion for a simple module to be a simple current. 
Lemma 4.2. Let $V$ be a simple, rational and $C_{2}$-cofinite vertex operator algebra of CFT type. Let $M^{0}, M^{1}, \cdots, M^{d}$ be as before with the corresponding conformal weights $\lambda_{i}>0$ for $0<i \leq d$. Then $0<\operatorname{qdim}_{V} M^{i}<\infty$ for any $0 \leq i \leq d$.

Proof. Since $V$ is rational and $C_{2}$-cofinite, we can use the modular transformation rule given in (2.2). By definition, we have

$$
\begin{aligned}
\operatorname{qdim}_{V} M^{i} & =\lim _{y \rightarrow 0} \frac{Z_{M^{i}}(i y)}{Z_{V}(i y)} \\
& =\lim _{\tau \rightarrow i \infty} \frac{Z_{M^{i}}\left(-\frac{1}{\tau}\right)}{Z_{V}\left(-\frac{1}{\tau}\right)} \\
& =\lim _{\tau \rightarrow i \infty} \frac{\sum_{j=0}^{d} S_{i, j} Z_{M^{j}}(\tau)}{\sum_{j=0}^{d} S_{0, j} Z_{M^{j}}(\tau)} \\
& =\lim _{q \rightarrow 0^{+}} \frac{\sum_{j=0}^{d} S_{i, j} \operatorname{ch}_{q} M^{j}}{\sum_{j=0}^{d} S_{0, j} \operatorname{ch}_{q} M^{j}} \\
& =\frac{S_{i, 0}}{S_{0,0}} .
\end{aligned}
$$

The last equality is true because the conformal weight $\lambda_{i}>0$ except $\lambda_{0}$, which implies $\lim _{q \rightarrow 0^{+}} \operatorname{ch}_{q} M^{j}=0$ for $0<j \leq d$. By Theorem 4.1 we know that $S_{i, 0} \neq 0$ for $0 \leq i \leq d$. So $\operatorname{qdim}_{V} M^{i}$ exists for all $0 \leq i \leq d$. By Remark 3.4 we conclude $0<\operatorname{qdim}_{V} M^{i}<\infty$.

Remark 4.3. The computation given in (4.1) indicates that the quantum dimension of a $V$-module, in some sense, only depends on the $S$-matrix. From Remark 2.13 we know that the $S$-matrix does not depend on the element $v$ we choose. Therefore we can actually define qdim $_{V} M$ in the following way:

$$
\operatorname{qdim}_{V} M=\lim _{y \rightarrow 0} \frac{Z_{M}(v, i y)}{Z_{V}(v, i y)}
$$

for any homogeneous $v \in V$ with $\left.o(v)\right|_{V_{0}} \neq 0$.

Remark 4.4. Lemma 4.2 can be generalized as follows. Assume $V$ is a simple, rational and $C_{2}$-cofinite VOA with $V \cong V^{\prime}$. Let $M^{i}$ be as before. Also assume $\lambda_{k}=\lambda_{\min }=\min _{i}\left\{\lambda_{i}\right\}$ and $\lambda_{j}>\lambda_{k} \forall j \neq k$. (It is not clear if the assumption that there is a unique $k$ such that $\lambda_{k}=\lambda_{\text {min }}$ is always satisfied for rational VOAs.) Then $\operatorname{qdim}_{V} M^{i}=\frac{S_{i, k}}{S_{0, k}}$. The same consideration also applies to several other results including Propositions 4.5, 4.17, and Theorem 5.10 below with suitable modifications.

By using the Verlinde formula, we get the following result about global dimensions.

Proposition 4.5. Let $V$ be as in Lemma 4.2; the global dimension of $V$ is given by $\operatorname{glob}(V)=\frac{1}{S_{0,0}^{2}}$.

Proof. By Lemma 4.2

$$
\operatorname{glob}(V)=\sum_{i=0}^{d}\left(\frac{S_{i, 0}}{S_{0,0}}\right)^{2}=\sum_{i=0}^{d} \frac{S_{i, 0}^{2}}{S_{0,0}^{2}}=\frac{\sum_{i=0}^{d} S_{i, 0}^{2}}{S_{0,0}^{2}} .
$$


By Theorem 4.1 (1) and (2), $\sum_{i=0}^{d} S_{i, 0}^{2}=1$ and the result follows.

Next, we turn our discussion to the quantum dimension of tensor product of two modules.

Definition 4.6. Let $V$ be a vertex operator algebra, and $M^{1}, M^{2}$ be two $V$ modules. A module $(W, I)$, where $I \in I_{V}\left(\begin{array}{c}W \\ M^{1} M^{2}\end{array}\right)$, is called a tensor product

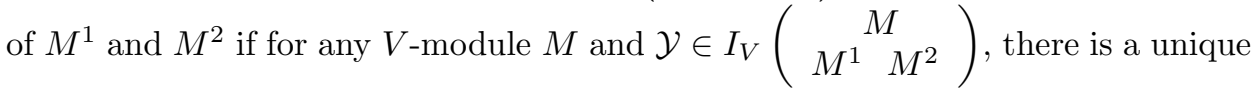
$V$-module homomorphism $f: W \rightarrow M$, such that $\mathcal{Y}=f \circ I$. As usual, we denote $(W, I)$ by $M^{1} \otimes M^{2}$.

Remark 4.7. It is well known that for a rational vertex operator algebra $V$, let $M^{0}, \cdots, M^{d}$ be all irreducible $V$-modules. Then $M^{i} \otimes M^{j}$ exists and

$$
M^{i} \otimes M^{j}=\sum_{k=0}^{d} N_{i, j}^{k} M^{k},
$$

where $N_{i, j}^{k}$ are the fusion rules.

Moreover, if $V$ is also $C_{2}$-cofinite, then the tensor product is commutative and associative and $N_{i, j}^{k}=N_{i, k^{\prime}}^{j^{\prime}}($ see [HL1]-[HL4], [Li2]).

Remark 4.8. It is easy to show that $V \otimes M=M$ for any $V$-module $M$.

Proposition 4.9. Let $V$ and $\left\{M^{0}, M^{1}, \cdots, M^{d}\right\}$ be as in Lemma 4.2, and also assume $V \cong V^{\prime}$. Then

$$
\operatorname{qdim}_{V}\left(M^{i} \otimes M^{j}\right)=\operatorname{qdim}_{V} M^{i} \cdot \operatorname{qdim}_{V} M^{j} .
$$

Proof. By Lemma 4.2 and Remark 4.7, it suffices to show

$$
\sum_{k=0}^{d} N_{i, j}^{k} \frac{S_{k, 0}}{S_{0,0}}=\frac{S_{i, 0}}{S_{0,0}} \frac{S_{j, 0}}{S_{0,0}} .
$$

Using Theorem 4.1 gives

$$
\begin{aligned}
\sum_{k=0}^{d} N_{i, j}^{k} \frac{S_{k, 0}}{S_{0,0}} & =\sum_{k, s=0}^{d} \frac{S_{i, s} S_{j, s} S_{s, k}^{-1}}{S_{0, s}} \frac{S_{k, 0}}{S_{0,0}} \\
& =\frac{1}{S_{0,0}} \sum_{s=0}^{d}\left(\frac{S_{i, s} S_{j, s}}{S_{0, s}} \sum_{k=0}^{d}\left(S_{s, k}^{-1} S_{k, 0}\right)\right) \\
& =\frac{1}{S_{0,0}} \sum_{s=0}^{d}\left(\frac{S_{i, s} S_{j, s}}{S_{0, s}} \delta_{s, 0}\right) \\
& =\frac{1}{S_{0,0}} \frac{S_{i, 0} S_{j, 0}}{S_{0,0}} \\
& =\frac{S_{i, 0}}{S_{0,0}} \frac{S_{j, 0}}{S_{0,0}} .
\end{aligned}
$$

The proof is complete.

Remark 4.10. Under the assumptions of Remark 4.4 one can easily prove that quantum dimensions are also multiplicative under tensor product using the Verlinde formula. 
We now turn our attention to the quantum dimensions of simple currents.

Definition 4.11. Let $V$ be a simple vertex operator algebra. A simple $V$-module $M$ is called a simple current if for any irreducible $V$-module $W, M \otimes W$ exists and is also a simple $V$-module.

It is clear from the definition that the simple current is an analogue of the one dimensional module for groups. Our goal is to establish that $M$ is a simple current if and only if $\operatorname{qdim}_{V} M=1$.

Remark 4.12. By Remark 4.8, if $V$ is a simple vertex operator algebra, then $V$ itself is a simple current.

Lemma 4.13. Let $V$ be a rational and $C_{2}$-cofinite simple vertex operator algebra, and $M, N$ be two admissible $V$-modules. Then $M \otimes N \neq 0$.

Proof. As $V$ is rational and $C_{2}$-cofinite, by Remark 4.7, we know the tensor product of any two $V$-modules exists. Thus we can consider

$$
(M \otimes N) \otimes N^{\prime}=M \otimes\left(N \otimes N^{\prime}\right) \supseteq M \otimes V=M \neq 0,
$$

which implies $M \otimes N \neq 0$.

Lemma 4.14. Let $V$ be a rational and $C_{2}$-cofinite simple vertex operator algebra of CFT type with $V \cong V^{\prime}$, and let $M^{0}, M^{1}, \cdots, M^{d}$ be as before with the corresponding conformal weights $\lambda_{i}>0,0<i \leq d$. Then for any irreducible $V$-module $M, \operatorname{qdim}_{V} M \geq 1$.

Proof. Let $M^{i}$ be an irreducible $V$-module with minimal quantum dimension. By Lemma 4.2, obviously $\mathrm{qdim}_{V} M^{i}>0$ and $\mathrm{qdim}_{V} M>0$. Then using Proposition 4.9, we get

$$
\operatorname{qdim}_{V} M^{i} \cdot \operatorname{qdim}_{V} M=\operatorname{qdim}_{V}\left(M^{i} \otimes M\right)>0 .
$$

$M^{i}$ was chosen to be of minimal quantum dimension, therefore

$$
\operatorname{qdim}_{V} M^{i} \cdot \operatorname{qdim}_{V} M \geq \operatorname{qdim}_{V} M^{i}>0 .
$$

Thus we get $\operatorname{qdim}_{V} M \geq 1$.

Lemma 4.15. Let $V$ be a rational and $C_{2}$-cofinite simple vertex operator algebra of CFT type with $V \cong V^{\prime}$, and let $M^{0}, M^{1}, \cdots, M^{d}$ be as before with the corresponding conformal weights $\lambda_{i}>0,0<i<d$. If $M$ is a simple current of $V$, then $\operatorname{qdim}_{V} M=1$.

Proof. Let $M$ be a simple current of $V$. Immediately we have $M \otimes M^{\prime}=V$. Then by Remark 3.5 and Proposition 4.9 we have

$$
1=q_{\operatorname{dim}_{V}} V=q_{\operatorname{dim}_{V}}\left(M \otimes M^{\prime}\right)=q_{\operatorname{dim}_{V}} M \cdot q_{\operatorname{dim}_{V}} M^{\prime}=\left(\operatorname{qdim}_{V} M\right)^{2} .
$$

This implies $\operatorname{qdim}_{V} M=1$.

Lemma 4.16. Let $V$ be a rational and $C_{2}$-cofinite simple vertex operator algebra, and $M^{0} \cong V, M^{1}, \cdots, M^{d}$ be as before. Fix an irreducible $V$-module $M^{s}$. Then for any $0 \leq i \leq d$ there exists $0 \leq j \leq d$ such that

$$
M^{i} \subseteq M^{s} \otimes M^{j} .
$$


Proof. Again, the rationality and $C_{2}$-cofiniteness guarantee that tensor products are well defined and associative. So

$$
M^{i} \subseteq\left(M^{s} \otimes M^{s^{\prime}}\right) \otimes M^{i}=M^{s} \otimes\left(M^{s^{\prime}} \otimes M^{i}\right),
$$

where $M^{s^{\prime}}$ is the dual of $M^{s}$. Note that $M^{s^{\prime}} \otimes M^{i}=\bigoplus_{j=0}^{d} N_{s^{\prime}, i}^{j} M^{j}$. Thus

$$
M^{i} \subseteq \bigoplus_{j=0}^{d} N_{s^{\prime}, i}^{j} M^{s} \otimes M^{j}
$$

Since $M^{i}$ is simple, $M^{i} \subseteq M^{s} \otimes M^{j}$ for some $j$.

Proposition 4.17. Let $V$ be a rational and $C_{2}$-cofinite simple vertex operator algebra of CFT type with $V \cong V^{\prime}$, and let $M^{0}, M^{1}, \cdots, M^{d}$ be as before with the corresponding conformal weights $\lambda_{i}>0,0<i \leq d$. Then a $V$-module $M$ is a simple current if and only if $\mathrm{qdim}_{V} M=1$.

Proof. By Lemma 4.15 any simple current has quantum dimension 1. Now assume $M$ is a $V$-module such that $\operatorname{qdim}_{V} M=1$. Obviously, Lemma 4.14 shows that $M$ is simple. Lemma 4.16 claims that

This implies

$$
\bigoplus_{i=0}^{d} M^{i} \subseteq \bigoplus_{i=0}^{d} M \otimes M^{i}
$$

$$
\operatorname{qdim}_{V}\left(\bigoplus_{i=0}^{d} M^{i}\right) \leq \operatorname{qdim}_{V} \bigoplus_{i=0}^{d} M \otimes M^{i}
$$

Since qdim $_{V} M=1$, computing the quantum dimensions on both sides of the above equation gives

$$
\operatorname{qdim}_{V}\left(\bigoplus_{i=0}^{d} M^{i}\right)=\sum_{i=0}^{d} 1 \cdot \operatorname{qdim}_{V} M^{i}=\sum_{i=0}^{d} \operatorname{qdim}_{V} M \otimes M^{i}=\operatorname{qdim}_{V}\left(\bigoplus_{i=0}^{d} M \otimes M^{i}\right)
$$

We obtain $\bigoplus_{i=0}^{d} M^{i}=\bigoplus_{i=0}^{d} M \otimes M^{i}$.

By Lemma 4.13, we can conclude that for any $M^{i}, i=0, \cdots, d$, there exists some $j=0, \cdots, d$, such that $M \otimes M^{i}=M^{j}$, i.e. $M$ is a simple current.

4.3. Examples. Now, by using the properties above we can compute more examples about quantum dimensions.

Example 4.18. Let $\mathfrak{g}$ be a finite dimensional simple Lie algebra with Cartan subalgebra $\mathfrak{h}$, and $\hat{\mathfrak{g}}$ be the corresponding affine Lie algebra. Fix a positive integer $k$. For any $\lambda \in \mathfrak{h}^{*}$, we denote the corresponding irreducible highest weight module for $\hat{\mathfrak{g}}$ by $L_{\mathfrak{g}}(k, \lambda)$. It is proved in [DL, [FZ, Li1] that $L_{\mathfrak{g}}(k, 0)$ is a rational simple vertex operator algebra and all irreducible $L_{\mathfrak{g}}(k, 0)$-modules are classified as $\left\{L_{\mathfrak{g}}(k, \lambda) \mid\langle\lambda, \theta\rangle \leq k, \lambda \in \mathfrak{h}^{*}\right.$ is a dominant integral weight $\}$, where $\theta$ is the longest root of $\mathfrak{g}$, and $(\theta, \theta)=2$.

The quantum dimensions of the irreducible $L_{\mathfrak{g}}(k, 0)$-modules can be computed using Lemma 4.2 and the modular transformation property of affine characters $[\mathrm{K}]$. The formula is given in [C]:

$$
\operatorname{qdim}_{L_{\mathfrak{g}}(k, 0)} L_{\mathfrak{g}}(k, \lambda)=\prod_{\alpha>0} \frac{\langle\lambda+\rho, \alpha\rangle_{q}}{\langle\rho, \alpha\rangle_{q}},
$$


where $\rho$ is the Weyl vector, $\alpha$ belongs to the set of positive roots and $n_{q}=\frac{q^{n}-q^{-n}}{q-q^{-1}}$, where $q=e^{i \pi /(k+\breve{h})}$ and $\breve{h}$ is the dual coxeter number of $\mathfrak{g}$. In the operator algebra framework, the statistic dimension of $L_{\mathfrak{g}}(k, \lambda)$ is given by the same formula when $\mathfrak{g}$ is of type $A$ in Wa.

Example 4.19. Let $V_{L}$ be the lattice vertex operator algebra associated to a positive definite even lattice $L$. It is proved in [D], DLM5] that $V_{L}$ is rational and $C_{2}$-cofinite, and $\left\{V_{L+\mu_{i}} \mid i \in L^{\circ} / L\right\}$ gives a complete list of all irreducible $V_{L^{-}}$ modules, where $L^{\circ}$ is the dual lattice of $L$. By $\left[\mathrm{DL}\right.$, every irreducible $V_{L}$-module is a simple current. Thus

$$
\operatorname{qdim}_{V_{L}} V_{L+\mu_{i}}=1
$$

by Proposition 4.17

This implies a well-known property on the theta functions of lattices. Note that $\operatorname{ch}_{q} V_{L+\mu_{i}}=\frac{\theta_{L+\mu_{i}}(q)}{\eta(q)^{d}}$, where $\theta_{L+\mu_{i}}(q)=\sum_{\alpha \in L+\mu_{i}} q^{(\alpha, \alpha) / 2}$ and $\eta(q)=q^{1 / 24} \prod_{n \geq 1}(1-$ $q^{n}$ ) and $d$ is the rank of $L$. Then

$$
\lim _{q \rightarrow 1} \frac{\theta_{L+\mu_{i}}(q)}{\theta_{L}(q)}=\lim _{q \rightarrow 1} \frac{\operatorname{ch}_{q} V_{L+\mu_{i}}}{\operatorname{ch}_{q} V_{L}}=1 .
$$

Example 4.20. We now consider the vertex operator algebra $L\left(\frac{1}{2}, 0\right)$ associated to the Virasoro algebra with central charge $\frac{1}{2}$. This is a rational and $C_{2}$-cofinite vertex operator algebra with only three irreducible modules $L\left(\frac{1}{2}, 0\right), L\left(\frac{1}{2}, \frac{1}{2}\right)$, and $L\left(\frac{1}{2}, \frac{1}{16}\right)$ (see [DLM5], [DMZ, [W]). We use two different methods to compute the quantum dimensions of the modules.

(1) We first compute the quantum dimensions by using the $S$-matrix (see Lemma 4.2). For short we use $Z_{0}(\tau), Z_{\frac{1}{2}}(\tau), Z_{\frac{1}{16}}(\tau)$ to denote $Z_{L\left(\frac{1}{2}, 0\right)}(\tau), Z_{L\left(\frac{1}{2}, \frac{1}{2}\right)}(\tau)$, and $Z_{L\left(\frac{1}{2}, \frac{1}{16}\right)}(\tau)$ respectively. The modular transformation rules below are given in [Ka]:

$$
\begin{aligned}
Z_{0}\left(-\frac{1}{\tau}\right) & =\frac{1}{2} Z_{0}(\tau)+\frac{1}{2} Z_{\frac{1}{2}}(\tau)+\frac{\sqrt{2}}{2} Z_{\frac{1}{16}}(\tau), \\
Z_{\frac{1}{2}}\left(-\frac{1}{\tau}\right) & =\frac{1}{2} Z_{0}(\tau)+\frac{1}{2} Z_{\frac{1}{2}}(\tau)-\frac{\sqrt{2}}{2} Z_{\frac{1}{16}}(\tau), \\
Z_{\frac{1}{16}}\left(-\frac{1}{\tau}\right) & =\frac{\sqrt{2}}{2} Z_{0}(\tau)-\frac{\sqrt{2}}{2} Z_{\frac{1}{2}}(\tau) .
\end{aligned}
$$

By Lemma 4.2, we get

$$
\begin{aligned}
\operatorname{qdim}_{L\left(\frac{1}{2}, 0\right)} L\left(\frac{1}{2}, 0\right) & =1, \\
\operatorname{qdim}_{L\left(\frac{1}{2}, 0\right)} L\left(\frac{1}{2}, \frac{1}{2}\right) & =\frac{1 / 2}{1 / 2}=1, \\
\operatorname{qdim}_{L\left(\frac{1}{2}, 0\right)} L\left(\frac{1}{2}, \frac{1}{16}\right) & =\frac{\sqrt{2} / 2}{1 / 2}=\sqrt{2} .
\end{aligned}
$$


(2) We can also compute the quantum dimensions by using Proposition 4.9. The fusion rules for these modules are well known (see [DMZ], [W]):

$$
\begin{aligned}
& \text { (1) } L\left(\frac{1}{2}, 0\right) \text { is the identity, } \\
& \text { (2) } L\left(\frac{1}{2}, \frac{1}{2}\right) \times L\left(\frac{1}{2}, \frac{1}{2}\right)=L\left(\frac{1}{2}, 0\right) \\
& \text { (3) } L\left(\frac{1}{2}, \frac{1}{2}\right) \times L\left(\frac{1}{2}, \frac{1}{16}\right)=L\left(\frac{1}{2}, \frac{1}{16}\right), \\
& \text { (4) } L\left(\frac{1}{2}, \frac{1}{16}\right) \times L\left(\frac{1}{2}, \frac{1}{16}\right)=L\left(\frac{1}{2}, 0\right)+L\left(\frac{1}{2}, \frac{1}{2}\right)
\end{aligned}
$$

Obviously, $L\left(\frac{1}{2}, 0\right)$ and $L\left(\frac{1}{2}, \frac{1}{2}\right)$ are simple currents, so we have

$$
\operatorname{qdim}_{L\left(\frac{1}{2}, 0\right)} L\left(\frac{1}{2}, 0\right)=\operatorname{qdim}_{L\left(\frac{1}{2}, 0\right)} L\left(\frac{1}{2}, \frac{1}{2}\right)=1 .
$$

Using (4.3) (4.4) and Proposition 4.9 we have

$$
\left[\operatorname{qdim}_{L\left(\frac{1}{2}, 0\right)} L\left(\frac{1}{2}, \frac{1}{16}\right)\right]^{2}=1+1=2 .
$$

That implies

$$
\operatorname{qdim}_{L\left(\frac{1}{2}, 0\right)} L\left(\frac{1}{2}, \frac{1}{16}\right)=\sqrt{2} .
$$

Example 4.21. Let $L(c, h)$ be the irreducible Virasoro algebra module as in Example 3.12, It is proved in $[\mathrm{W}$ that the vertex operator algebra $L(c, 0)$ is rational if and only if $c=c_{p, q}=1-\frac{6(p-q)^{2}}{p q}$, where $p, q \in\{2,3,4, \cdots\}$, and $p, q$ are relatively prime. Also, all irreducible $L\left(c_{p, q}, 0\right)$-modules are classified as $L\left(c_{p, q}, h_{m, n}\right)$ for $0<m<p, 0<n<q$, where $h_{m, n}=\frac{(n p-m q)^{2}-(p-q)^{2}}{4 p q}$. The fusion rules are also determined in $[\mathrm{W}]$.

Let $\chi_{m, n}^{p, q}(\tau)$ denote the character of $L\left(c_{p, q}, h_{m, n}\right)$. The $S$-modular transformation of characters has the following form [CIZ1, [CIZ2, [IZ:

$$
\chi_{m, n}^{p, q}(-1 / \tau)=\sum_{m^{\prime}, n^{\prime}} S_{m, n}^{m^{\prime}, n^{\prime}} \chi_{m^{\prime}, n^{\prime}}^{p, q}(\tau)
$$

where

$$
S_{m, n}^{m^{\prime}, n^{\prime}}=\sqrt{\frac{8}{p q}}(-1)^{m^{\prime} n+n^{\prime} m+1} \sin \left(\frac{\pi m m^{\prime} q}{p}\right) \sin \left(\frac{\pi n n^{\prime} p}{q}\right) .
$$

The case $p=q-1$ gives the unitary discrete series, which has been extensively studied in physics and conformal nets theory. Using Lemma 4.2 and formula (4.5) one can easily compute the quantum dimensions of irreducible modules for the unitary discrete series. For the nonunitary case, $\lambda_{\min }=\frac{1-(p-q)^{2}}{4 p q} \neq 0$ (see [DM3]). In this case, one needs to find $m, n$ such that $|m q-n p|=1$. We only discuss two special cases $(p, q)=(2,5)$ and $(3,5)$ here. The quantum dimensions of their irreducible modules are computed using the $S$ matrix and Remark 4.4.

1) The case $(p, q)=(2,5): c_{p, q}=-\frac{22}{5}$ and $L\left(c_{2,5}, 0\right)$ has 2 irreducible modules $L\left(c_{2,5}, h_{1, n}\right), n=1,2$. Here $h_{1,1}=0$, and $h_{1,2}=-\frac{1}{5}=\lambda_{\min }$. A straightforward calculation gives

$$
S_{1,1}^{1,2}=\sqrt{\frac{4}{5}} \sin \left(\frac{4 \pi}{5}\right), S_{1,2}^{1,2}=-\sqrt{\frac{4}{5}} \sin \left(\frac{8 \pi}{5}\right) .
$$


Thus

$$
\operatorname{qdim}_{L\left(c_{2,5}, 0\right)} L\left(c_{2,5}, h_{1,1}\right)=1,
$$

and

$$
\operatorname{qdim}_{L\left(c_{2,5}, 0\right)} L\left(c_{2,5}, h_{1,2}\right)=\frac{S_{1,2}^{1,2}}{S_{1,1}^{1,2}}=-\frac{\sqrt{\frac{4}{5}} \sin \left(\frac{8 \pi}{5}\right)}{\sqrt{\frac{4}{5}} \sin \left(\frac{4 \pi}{5}\right)}=2 \cos \frac{\pi}{5} .
$$

2) The case $(p, q)=(3,5): c_{p, q}=-\frac{3}{5}$ and $L\left(c_{3,5}, 0\right)$ has 4 irreducible modules $L\left(c_{3,5}, h_{1, n}\right), n=1,2,3,4$. Here $h_{1,1}=0, h_{1,2}=-\frac{1}{20}=\lambda_{\min }, h_{1,3}=\frac{1}{5}$ and $h_{1,4}=\frac{3}{4}$. By using (4.5), it is easy to get

$$
\begin{aligned}
& S_{1,1}^{1,2}=\sqrt{\frac{8}{15}} \sin \left(\frac{5 \pi}{3}\right) \sin \left(\frac{6 \pi}{5}\right), \\
& S_{1,2}^{1,2}=-\sqrt{\frac{8}{15}} \sin \left(\frac{5 \pi}{3}\right) \sin \left(\frac{12 \pi}{5}\right), \\
& S_{1,3}^{1,2}=\sqrt{\frac{8}{15}} \sin \left(\frac{5 \pi}{3}\right) \sin \left(\frac{18 \pi}{5}\right), \\
& S_{1,4}^{1,2}=-\sqrt{\frac{8}{15}} \sin \left(\frac{5 \pi}{3}\right) \sin \left(\frac{24 \pi}{5}\right) .
\end{aligned}
$$

This implies

$$
\begin{aligned}
& \operatorname{qdim}_{L\left(c_{3,5}, 0\right)} L\left(c_{3,5}, h_{1,1}\right)=\operatorname{qdim}_{L\left(c_{3,5}, 0\right)} L\left(c_{3,5}, h_{1,4}\right)=1, \\
& \operatorname{qdim}_{L\left(c_{3,5}, 0\right)} L\left(c_{3,5}, h_{1,2}\right)=\frac{S_{1,2}^{1,2}}{S_{1,1}^{1,2}}=2 \cos \left(\frac{\pi}{5}\right), \\
& \operatorname{qdim}_{L\left(c_{3,5}, 0\right)} L\left(c_{3,5}, h_{1,3}\right)=\frac{S_{1,3}^{1,2}}{S_{1,1}^{1,2}}=2 \cos \left(\frac{\pi}{5}\right) .
\end{aligned}
$$

In the following example, the vertex operator algebra $V$ is neither rational nor $\mathrm{C}_{2}$-cofinite. But the quantum dimensions of its modules are still multiplicative under tensor product.

Example 4.22. Recall $L(1,0)$ and $L(1, h)$ from Example 3.12 The fusion rules for irreducible $L(1,0)$-modules are given in [M] and [DJ]. Assume $m, n \in \mathbb{Z}_{+}$and $m \geq n$; then

$$
L\left(1, m^{2}\right) \otimes L\left(1, n^{2}\right)=\bigoplus_{k=m-n}^{m+n} L\left(1, k^{2}\right) .
$$

By Example 3.12, we have

$$
\begin{aligned}
& \operatorname{qdim}_{L(1,0)}\left(L\left(1, m^{2}\right) \otimes L\left(1, n^{2}\right)\right) \\
= & \operatorname{qdim}_{L(1,0)}\left(\bigoplus_{k=m-n}^{m+n} L\left(1, k^{2}\right)\right) \\
= & \sum_{k=m-n}^{m+n}(2 k+1) \\
= & (2 m+1)(2 n+1) \\
= & \operatorname{qdim}_{L(1,0)} L\left(1, m^{2}\right) \cdot \operatorname{qdim}_{L(1,0)} L\left(1, n^{2}\right) .
\end{aligned}
$$


From the fusion rules, the only simple current among $L\left(1, m^{2}\right)$ is $L(1,0)$, which has quantum dimension 1.

By Example 4.22, it seems that even for vertex operator algebras that are not rational, Propositions 4.9 and 4.17 are still true. But the proofs of these propositions require that $V$ is rational and $C_{2}$-cofinite as the modularity of trace functions are used. This gives us a good reason to believe that there might be an alternating definition for quantum dimensions which does not involve the trace functions. We believe that the other two limits given in Proposition 3.6 are in the right direction.

\section{Possible values of Quantum dimensions}

In this section we will give a result on possible values of quantum dimensions using graph theory and the Perron-Frobenius Theorem. It turns out that the values of quantum dimensions are closely related to the possible values of the index of subfactors [J].

Definition 5.1. Let $A$ be an $n \times n$ matrix over $\mathbb{R}$, and $\lambda_{1}, \cdots, \lambda_{n}$ be all the eigenvalues of $A$. Then its spectral radius $\rho(A)$ is defined as

$$
\rho(A)=\max _{i}\left(\left|\lambda_{i}\right|\right) .
$$

The next result is part of the Perron-Frobenius Theorem (cf. $[\mathrm{BH}]$ ).

Theorem 5.2. Let $A=\left(a_{i j}\right)$ be an $n \times n$ positive matrix: $a_{i j}>0$, for $1 \leq i, j \leq n$. Then the following statements hold:

(1) There is a positive real number $r$, such that $r$ is an eigenvalue of $A$ and any other eigenvalue $\lambda$ (possibly complex) is strictly smaller than $r$ in absolute value, $|\lambda|<$ r, i.e. $\rho(A)=r$.

(2) There exists an eigenvector $v=\left(v_{1}, \cdots, v_{n}\right)$ of $A$ with eigenvalue $r$ such that all components of $v$ are positive.

(3) There are no other positive (moreover nonnegative) eigenvectors except positive multiples of $v$, i.e. all other eigenvectors must have at least one negative or nonreal component.

Remark 5.3. In the case that $A$ is nonnegative, we can use positive matrices to approach $A$. So if there is a positive eigenvector of $A$ with positive eigenvalue $r$, then $r=\rho(A)$.

Remark 5.4. Let $V$ be a simple vertex operator algebra as in Lemma 4.2 and $M^{i}$ for $i=0, \cdots, d$ be the irreducible $V$-modules as before. Theorem 4.1 and Lemma 4.2 assert that $\operatorname{qdim}_{V} M^{i}$ is a positive eigenvalue of $N(i)$ (defined in the next lemma) with eigenvector $v=\left(\frac{S_{0,0}}{S_{0,0}}, \frac{S_{0,1}}{S_{0,0}}, \cdots, \frac{S_{0, d}}{S_{0,0}}\right)^{T}$ whose entries are all positive (since each component is a quantum dimension). By Remark 5.3, one knows that $\operatorname{qdim}_{V} M^{i}$ is exactly the spectral radius of $N(i)$, i.e. $\operatorname{qdim}_{V}\left(M^{i}\right)=\rho(N(i))$.

The following lemmas are devoted to proving the main result of this section.

Lemma 5.5. Let $V$ be a vertex operator algebra as in Lemma 4.2, and $N(i)=$ $\left(N_{i, j}^{k}\right)_{j, k}$ be the fusion matrix. Then $N(i)^{T}=N\left(i^{\prime}\right)$. 
Proof. Using Theorem 4.1 gives

$$
\begin{aligned}
N_{i, j}^{k} & =\sum_{s=0}^{d} \frac{S_{j, s} S_{i, s} S_{s, k}^{-1}}{S_{0, s}} \\
& =\sum_{s^{\prime}=0}^{d} \frac{S_{j^{\prime}, s^{\prime}} S_{i^{\prime}, s^{\prime}} S_{s^{\prime}, k^{\prime}}^{-1}}{S_{0, s^{\prime}}} \\
& =\sum_{s=0}^{d} \frac{S_{j^{\prime}, s} S_{i^{\prime}, s} S_{s, k^{\prime}}^{-1}}{S_{0, s}} \\
& =N_{i^{\prime}, j^{\prime}}^{k^{\prime}}=N_{i^{\prime}, k}^{j} .
\end{aligned}
$$

The proof is complete.

Since the fusion algebra is commutative, the following corollary is now obvious.

Corollary 5.6. The fusion matrix $N(i)$ is normal, i.e. $N(i)^{T} N(i)=N(i) N(i)^{T}$.

Lemma 5.7. The matrix $\left(\begin{array}{cc}0 & N(i) \\ N(i)^{T} & 0\end{array}\right)$ is a symmetric matrix whose spectral radius equals $\operatorname{qdim}_{V} M^{i}$.

Proof. It is clear that $\bar{N}(i)=\left(\begin{array}{cc}0 & N(i) \\ N(i)^{T} & 0\end{array}\right)$ is a symmetric matrix. Also, the matrices $N(i)$ and $N(i)^{T}=N\left(i^{\prime}\right)$ have the same spectral radius qdim $M^{i}$ with the same eigenvector $v$ (Remark [5.4). Thus the vector $\left(\begin{array}{l}v \\ v\end{array}\right)$ is an eigenvector of $\bar{N}(i)$ with eigenvalue $\operatorname{qdim}_{V} M^{i}$. Again by Remark 5.3, $\rho(\bar{N}(i))=\operatorname{qdim}_{V} M^{i}$.

In graph theory, the adjacency matrix of a finite graph $G$ on $n$ vertices is the $n \times n$ matrix where the nondiagonal entry $a_{i j}$ is the number of edges from vertex $i$ to vertex $j$, and the diagonal entry $a_{i i}$ is the number of loops from vertex $i$ to itself. Thus any symmetric matrix with all entries nonnegative integers is the adjacency matrix of a certain finite graph.

Definition 5.8. Let $G$ be a graph and $M$ be its adjacency matrix. The spectral norm of $G$ is defined as the spectral radius of its adjacency matrix $M$, which is denoted by $\|G\|$.

Remark 5.9. If $G_{i}, i=1, \cdots, k$, are all the connected subgraphs of $G$, then

$$
\max _{i}\left\|G_{i}\right\|=\|G\| \text {. }
$$

Theorem 5.10. Let $V$ be a simple vertex operator algebra and $\left\{M^{0}, \cdots, M^{d}\right\}$ the inequivalent irreducible $V$-modules as in Lemma 4.2. Then for any $0 \leq i \leq d$,

$$
\operatorname{qdim}_{V} M^{i} \in\{2 \cos (\pi / n) \mid n \geq 3\} \cup\{a \mid 2 \leq a<\infty, \text { a is algebraic }\} .
$$

Proof. Since $\operatorname{qdim}_{V} M^{i}$ is the eigenvalue of the fusion matrix $N(i)$ whose entries are all nonnegative integers, it is an algebraic number.

By Lemma 5.7, we know that the matrix

$$
\bar{N}(i)=\left(\begin{array}{cc}
0 & N(i) \\
N(i)^{T} & 0
\end{array}\right)
$$


is symmetric with all entries nonnegative integers. Thus $\bar{N}(i)$ is the adjacency matrix of a certain graph, say, $G_{i}$, whose norm is actually the quantum dimension of $M^{i}$.

If $\left\|G_{i}\right\| \geq 2$, we are done. If $\left\|G_{i}\right\|<2$, it follows from [S] and [LS] that each connected subgraph of $G_{i}$ is of $A D E$ type. Also, the spectral norms of $A D E$ type graphs are of the form $2 \cos (\pi / n)$ with $n \geq 3$.

Remark 5.11. The possible values for the index of subfactors given in [J] are

$$
\left\{4 \cos ^{2}(\pi / n) \mid n \geq 3\right\} \cup[4, \infty) .
$$

So the possible values for quantum dimensions given in the previous theorem are exactly the square root of index of subfactors but restricted to algebraic numbers.

Remark 5.12. The possible values that are less than 2 can be realized. Take $\mathfrak{g}=$ $s l_{2}(\mathbb{C})$, and let $\lambda$ be the fundamental weight. Consider different levels $k \in \mathbb{Z}_{+}$. By Example 4.18, one easily sees that

$$
\operatorname{qdim}_{L_{\mathfrak{g}}(k, 0)} L_{\mathfrak{g}}(k, \lambda)=2 \cos \left(\frac{\pi}{k+2}\right), k \geq 1 .
$$

But we do not know which number greater than or equal to 2 can be realized as a quantum dimension. According to [ENO, the quantum dimensions for rational and $C_{2}$-cofinite vertex operator algebras are cyclotomic integers.

\section{Quantum Galois theory}

In this section, we study the Galois theory for vertex operator algebras. In classical Galois theory, we need the degree $[E: F]$ of a field $E$ over a subfield $F$, which is defined as $\operatorname{dim}_{F} E$. To have a Galois theory for vertex operator algebras, we also need to define the degree of a vertex operator algebra $V$ over a vertex operator subalgebra $U$. We define the degree $[V: U]$ to be the quantum $\operatorname{dimension} \operatorname{qdim}_{U} V$. In fact, establishing a quantum Galois theory is one of the main motivations for us to study the quantum dimensions.

We need the following theorems for further discussions.

Theorem 6.1. Let $V$ be a simple vertex operator algebra, $G$ be a compact subgroup of $\operatorname{Aut}(V)$, and $G$ acts continuously on $V$. Then as a $\left(G, V^{G}\right)$-module, $V=\bigoplus_{\chi \in \operatorname{Irr}(G)}\left(W_{\chi} \otimes V_{\chi}\right)$, where

(1) $V_{\chi} \neq 0, \forall \chi \in \operatorname{Irr}(G)$,

(2) $V_{\chi}$ is an irreducible $V^{G}$-module, $\forall \chi \in \operatorname{Irr}(G)$,

(3) $V_{\chi} \cong V_{\lambda}$ as $V^{G}$-modules if and only if $\chi=\lambda$.

Theorem 6.2. Let $V$ be as in the previous theorem, and $G$ be a finite subgroup of $\operatorname{Aut}(V)$. Then the map $H \rightarrow V^{H}$ gives a bijection between subgroups of $G$ and subVOAs of $V$ containing $V^{G}$.

Theorem 6.1 is established in DLM1. If $G$ is solvable, this result has been obtained previously in DM1. Theorem 6.2 is given in [DM1 and [HMT].

It is well known in classical Galois theory that if $G$ is a finite automorphism group of field $E$, then $\left[E: E^{G}\right]=o(G)$. The next result is a vertex operator algebra analogue of this result. 
Theorem 6.3. Let $V$ be a rational and $C_{2}$-cofinite simple vertex operator algebra. Also assume that $V$ is $g$-rational and the conformal weight of any irreducible $g$ twisted $V$-module is positive except for $V$ itself for all $g \in G$. Then $\left[V: V^{G}\right]$ exists and equals $o(G)$.

Proof. It is well known that

$$
o(G)=\sum_{\chi \in \operatorname{Irr}(G)}\left(\operatorname{dim} W_{\chi}\right)^{2}
$$

By Theorem 6.1] if $\operatorname{qdim}_{V^{G}} V_{\chi}$ exists for all $\chi \in \operatorname{Irr}(G)$, then $\left[V: V^{G}\right]$ exists and is equal to $\sum_{\chi \in \operatorname{Irr}(G)} \operatorname{dim} W_{\chi} \cdot \operatorname{qdim}_{V^{G}} V_{\chi}$. The theorem holds if $\operatorname{qdim}_{V^{G}} V_{\chi}=\chi(1)=$ $\operatorname{dim} W_{\chi}$.

By the orthogonality of characters of representations of a finite group, we notice that $\operatorname{ch}_{q}\left(V_{\chi}\right)=\frac{1}{o(G)} \sum_{g \in G} Z_{V}(1, g, q) \overline{\chi(g)}$. By Theorem 2.12 we have

$$
\begin{aligned}
\operatorname{qdim}_{V^{G}} V_{\chi} & =\lim _{q \rightarrow 1^{-}} \frac{\sum_{g \in G} Z_{V}(1, g, q) \overline{\chi(g)}}{\sum_{g \in G} Z_{V}(1, g, q)} \\
& =\lim _{\tau \rightarrow i \infty} \frac{\sum_{g \in G} Z_{V}\left(1, g,-\frac{1}{\tau}\right) \overline{\chi(g)}}{\sum_{g \in G} Z_{V}\left(1, g,-\frac{1}{\tau}\right)} \\
& =\lim _{\tau \rightarrow i \infty} \frac{\sum_{g \in G, N_{i} \in \mathscr{M}(g)} S_{0, i}(1, g) Z_{N_{i}}(g, 1, \tau) \overline{\chi(g)}}{\sum_{g \in G, N_{i} \in \mathscr{M}(g)} S_{0, i}(1, g) Z_{N_{i}}(g, 1, \tau)} \\
& =\lim _{q \rightarrow 0^{+}} \frac{\sum_{g \in G, N_{i} \in \mathscr{M}(g)} S_{0, i}(1, g) Z_{N_{i}}(g, 1, q) \overline{\chi(g)}}{\sum_{g \in G, N_{i} \in \mathscr{M}(g)} S_{0, i}(1, g) Z_{N_{i}}(g, 1, q)} .
\end{aligned}
$$

By the assumption, we know that

$$
\lim _{q \rightarrow 0^{+}} q^{c / 24} Z_{V}(1,1, q) \neq 0
$$

and

$$
\lim _{q \rightarrow 0^{+}} q^{c / 24} Z_{N_{i}}(g, 1, q)=0
$$

for any other $N_{i}$. This implies that

$$
\operatorname{qdim}_{V^{G}} V_{\chi}=\chi(1)=\operatorname{dim} W_{\chi},
$$

and the theorem follows.

Remark 6.4. In Theorem 6.3, under certain conditions, we proved for a finite group $G<\operatorname{Aut}(V), \operatorname{qdim}_{V^{G}} V_{\chi}=\chi(1)$. It seems that this result is still true for a compact group $G<\operatorname{Aut}(V)$ as assumed in DLM1. Here are some examples.

Example 6.5. Let $V_{L}$ be the lattice VOA associated to a positive definite even lattice $L$ of rank $d$ with a nondegenerate bilinear form ( , ). Set $\mathfrak{h}=L \otimes_{\mathbb{Z}} \mathbb{R}$ and let $M(1)$ be the rank $d$ Heisenberg vertex operator algebra associated to $\mathfrak{h}$. Then $V_{L} \cong M(1) \otimes\left(\bigoplus_{\alpha \in L} \mathbb{C} e^{\alpha}\right)$ as linear spaces. Recall that $L^{\circ}$ is the dual lattice of $L$. Then $L \otimes_{\mathbb{Z}} \mathbb{R} / L^{\circ} \cong T^{n}$ is a compact Lie group acting continuously on $V_{L}$ in the following way: for any $\beta \in \mathfrak{h}$,

$$
\begin{aligned}
e^{2 \pi i \beta(0)}: V_{L} & \rightarrow V_{L}, \\
a \otimes e^{\alpha} & \mapsto e^{2 \pi i(\beta, \alpha)} a \otimes e^{\alpha} .
\end{aligned}
$$


Since $\beta(0)$ is a derivation of $V_{L}, e^{2 \pi i \beta(0)}$ is an automorphism of $V_{L}$ such that $e^{2 \pi i \beta(0)}=1$ if $\beta \in L^{\circ}$. As a result, the torus $T^{n}$ is a compact subgroup of $\operatorname{Aut}\left(V_{L}\right)$. It is easy to see that $\left(V_{L}\right)^{T^{n}}=M(1)$. By Theorem 6.1 we have a decomposition of $V_{L}$ :

$$
V_{L}=\bigoplus_{\alpha \in L} M(1, \alpha) \otimes \mathbb{C} e^{\alpha},
$$

where $M(1, \alpha)$ is an $M(1)$-module with weight $\alpha$, and $\mathbb{C} e^{\alpha}$ is an irreducible $T^{n_{-}}$ module. We have already known from Example 3.11 that $\operatorname{qdim}_{M(1)} M(1, \alpha)=1$. That is, $\operatorname{qdim}_{M(1)} M(1, \alpha)=\operatorname{dim} \mathbb{C} e^{\alpha}$.

Example 6.6. Let $V=V_{L}$ be the lattice vertex operator algebra associated with the root lattice $L=\mathbb{Z} \alpha$ of type $A_{1}$ where $(\alpha, \alpha)=2$. It is well known that $S O(3)$ is a subgroup of $\operatorname{Aut}(V)$ and we have the following decomposition DG]:

$$
V=\bigoplus_{m \geq 0} W_{2 m} \otimes L\left(1, m^{2}\right)
$$

where $L\left(1, m^{2}\right)$ is the highest weight module for the Virasoro VOA $L(1,0)$ with highest weight $m^{2}$, and $W_{2 m}$ is the irreducible $2 m+1$ dimensional highest weight module for $S O(3)$ with highest weight $m$. In particular, $V^{S O(3)}=L(1,0)$. By Example 4.22, one gets

$$
\operatorname{dim} W_{2 m}=\operatorname{qdim}_{L(1,0)} L\left(1, m^{2}\right)=2 m+1 .
$$

Motivated by these two examples, we make the following conjecture:

Conjecture 6.7. Let $V=(V, Y, 1, \omega)$ be a rational and $C_{2}$-cofinite simple vertex operator algebra, and $G$ be a subgroup of $\operatorname{Aut}(V)$. Assume that $G$ is a finitedimensional compact Lie group which acts on $V$ continuously. Then the decomposition

$$
V=\sum_{\chi \in \operatorname{Irr}(G)} W_{\chi} \otimes V_{\chi}
$$

has the following property:

$$
\operatorname{dim} W_{\chi}=\operatorname{qdim}_{V^{G}} V_{\chi} .
$$

We now turn our attention to the Galois extensions in the theory of vertex operator algebra. We need some definitions first.

Definition 6.8. Let $U$ be a vertex operator subalgebra of $V$ with the same Virasoro element. $V$ is called a Galois extension of $U$ if there exists a finite group $G<\operatorname{Aut}(V)$ such that $U=V^{G}$, and $\operatorname{qdim}_{U} V \leq o(G)$.

For any VOA extension $V \supset U$ we can define the Galois group $\operatorname{Gal}(V / U)=\{g \in$ $\left.\operatorname{Aut}(V)|g|_{U}=I d\right\}$ as in classical field theory. The following two theorems are our main results about Galois extensions.

Theorem 6.9. Let $V$ be a simple vertex operator algebra, and $G<\operatorname{Aut}(V)$ a finite group. Then

$$
\operatorname{Gal}\left(V / V^{G}\right)=G
$$


Proof. Obviously $G \subset \operatorname{Gal}\left(V / V^{G}\right)$. We now prove the containment $\operatorname{Gal}\left(V / V^{G}\right) \subset$ $G$ with the help of Hopf algebra.

Let $\mathbb{C}[G]$ be the group algebra associated to $G$. Then $\mathbb{C}[G]$ is a cocommutative Hopf algebra with comultiplication $\Delta$, counit $\varepsilon$ and antipode $S$ :

$$
\begin{aligned}
& \Delta: \mathbb{C}[G] \rightarrow \mathbb{C}[G] \otimes \mathbb{C}[G], \Delta(g)=g \otimes g, \text { for } g \in G, \\
& \varepsilon: \mathbb{C}[G] \rightarrow \mathbb{C}, \varepsilon(g)=1, \text { for } g \in G, \\
& S: \mathbb{C}[G] \rightarrow \mathbb{C}[G], S(g)=g^{-1}, \text { for } g \in G .
\end{aligned}
$$

Recall that an element $g \in \mathbb{C}[G]$ with $\Delta(g)=g \otimes g$ and $\varepsilon(g)=1$ is called a grouplike element. It is well known that the set of group-like elements of $\mathbb{C}[G]$ is exactly $G$ itself. So it is enough to show that any $g \in \operatorname{Gal}\left(V / V^{G}\right)$ is a group-like element.

Since $\left.g\right|_{V G}=I d, g: V \rightarrow V$ gives a $V^{G}$-module homomorphism. Thus $g W \chi \subseteq$ $W \chi$, for any $\chi \in \operatorname{Irr}(G)$, where $W_{\chi}$ is the same as in Theorem 6.1. That implies

$$
g \in \bigoplus_{\chi \in \operatorname{Irr}(G)} \operatorname{End}\left(W_{\chi}\right)
$$

Together with the fact that $\mathbb{C}[G] \cong \bigoplus_{\chi \in \operatorname{Irr}(G)} \operatorname{End}\left(W_{\chi}\right), g$ can be viewed as an element in $\mathbb{C}[G]$. We write $g=\sum_{h \in G} \lambda_{h} h, \lambda_{h} \in \mathbb{C}$. As $\left.g\right|_{V G}=I d=\left.\sum_{h \in G} \lambda_{h} h\right|_{V^{G}}=$ $\sum_{h \in G} \lambda_{h} I d$, we get $\sum_{h \in G} \lambda_{h}=1$, i.e. $\varepsilon(g)=1$.

Now in order to show $\Delta(g)=g \otimes g$, it suffices to show that for any $\chi, \gamma \in \operatorname{Irr}(G)$, $g(a \otimes b)=g a \otimes g b$, where $a \in W_{\chi}$ and $b \in W_{\gamma}$. Let $W_{\chi}, W_{\gamma}$ be two $G$-submodules in $V$. It is proved in DM2 that there is a $G$-module isomorphism for sufficiently small $n$ :

$$
\begin{aligned}
\psi_{n}: W_{\chi} \otimes W_{\gamma} & \rightarrow\left\langle\sum_{m=n}^{\infty} u_{m} v \mid u \in W_{\chi}, v \in W_{\gamma}\right\rangle, \\
u \otimes v & \mapsto \sum_{m=n}^{\infty} u_{m} v .
\end{aligned}
$$

Since $g$ is an automorphism of $V, g\left(\sum_{m=n}^{\infty} u_{m} v\right)=\sum_{m=n}^{\infty}(g u)_{m} g v=\psi_{n}(g u \otimes g v)$. We get $g(u \otimes v)=g u \otimes g v$. Thus $g$ is a group-like element and the proof is complete.

Theorem 6.10. Let $V$ be a simple vertex operator algebra as in Theorem 6.3 , and $G$ a finite automorphism group of $V$. Then $H \mapsto V^{H}$ gives a one-to-one correspondence from the subgroups of $G$ and the vertex operator subalgebras of $V$ containing $V^{G}$ satisfying the following:

(1) For any subgroup $H$ of $G,\left[V: V^{H}\right]=o(H)$ and $\left[V^{H}: V^{G}\right]=[G: H]$,

(2) $H \triangleleft G$ if and only if $V^{H}$ is a Galois extension of $V^{G} \cdot \operatorname{Gal}\left(V^{H} / V^{G}\right) \cong G / H$ in this case

Proof. The one-to-one correspondence is given in Theorem 6.2. By Theorem 6.3 with $G$ replaced by $H$ we easily see that

$$
\left[V: V^{H}\right]=\sum_{\chi \in \operatorname{Irr}(H)} \operatorname{dim}\left(W_{\chi}\right) \cdot \chi(1)=\sum_{\chi \in \operatorname{Irr}(H)} \chi(1)^{2}=o(H) .
$$

Also, $\left[V^{H}: V^{G}\right]=\left[V: V^{G}\right] /\left[V: V^{H}\right]=[G: H]$, and this proves (1). 
For (2), we first notice that $g V^{H}=V^{g H g^{-1}}$ for $g \in G$. If $H \triangleleft G, G / H$ acts naturally on $V^{H}$. So $G / H$ is a subgroup of $\operatorname{Gal}\left(V^{H} / V^{G}\right)$. It is clear that $V^{G}=$ $\left(V^{H}\right)^{G / H}$. Then by Theorem 6.9, $\operatorname{Gal}\left(V^{H} / V^{G}\right) \cong G / H$. Together with the fact that $\left[V^{H}: V^{G}\right]=[G: H]$, we conclude that $V^{H}$ is a Galois extension of $V^{G}$.

Now we assume that $V^{H}$ is a Galois extension of $V^{G}$. For short we set $G^{\prime}=$ $\operatorname{Gal}\left(V^{H} / V^{G}\right)$. By part (1) and the definition of Galois extension we know that $\left(V^{H}\right)^{G^{\prime}}=V^{G}$ and

$$
[G: H]=\left[V^{H}: V^{G}\right]=\left[V^{H}:\left(V^{H}\right)^{G^{\prime}}\right] \leq o\left(G^{\prime}\right) .
$$

By Theorem 6.1, we have two decompositions,

$$
\begin{gathered}
V=\bigoplus_{\chi \in \operatorname{Irr}(G)} W_{\chi} \otimes V_{\chi}, \\
V^{H}=\bigoplus_{\chi \in \operatorname{Irr}(G)} R_{\chi} \otimes V_{\chi},
\end{gathered}
$$

where $R_{\chi} \subseteq W_{\chi}$ is a subspace, and each $R_{\chi}$ is an irreducible $G^{\prime}$-module. We also know every irreducible $G^{\prime}$-module occurs in $V^{H}$ by Theorem 6.1,

By Theorem 6.3 and equation (6.2), we have

$$
\begin{aligned}
{\left[V^{H}: V^{G}\right] } & =\sum_{\chi \in \operatorname{Irr}(G)} \operatorname{dim} R_{\chi} \cdot \operatorname{qdim}_{V^{G}} V_{\chi} \\
& =\sum_{\chi \in \operatorname{Irr}(G)} \operatorname{dim} R_{\chi} \cdot \operatorname{dim} W_{\chi} \\
& \geq \sum_{\chi \in \operatorname{Irr}(G)}\left(\operatorname{dim} R_{\chi}\right)^{2} \\
& =o\left(G^{\prime}\right) .
\end{aligned}
$$

Together with equation (6.1), we have that for any $\chi \in \operatorname{Irr}(G)$ with $R_{\chi} \neq 0$, $\operatorname{dim} R_{\chi}=\operatorname{qdim}_{V^{G}} V_{\chi}$, i.e. either $R_{\chi}=0$ or $R_{\chi}=W_{\chi}$, therefore for any $g \in G$, $g V^{H}=V^{g H g^{-1}} \subset V^{H}$. By Theorem 6.2 $H \leqslant g H g^{-1}$, which implies $H=g H g^{-1}$. The proof is complete.

Remark 6.11. Let $E \supset F$ be two fields. In classical Galois theory, the following two definitions for a Galois extension are equivalent:

(1) $E$ is called a Galois extension of $F$ if $F=E^{G}$ for some $G$, where $G$ is a finite subgroup of $\operatorname{Aut}(E)$,

(2) $E$ is called a Galois extension of $F$ if $\operatorname{dim}_{F} E=\operatorname{Gal}(E: F)$.

We believe that the same is true for vertex operator algebra. But we cannot prove it in this paper. However, if $V$ is a rational vertex operator algebra satisfying the assumptions given in Theorem 6.3, these two definitions are equivalent. Since $V^{H}$ in Theorem 6.10 does not satisfy the assumptions of Theorem 6.3 (these assumptions should hold according to conjectures in orbifold theory but have not been established), we need to use both (1) and (2) in the definition of Galois extension for vertex operator algebra to have a good Galois theory. 


\section{REFERENCES}

[ABD] T. Abe, G. Buhl and C. Dong, Rationality, Regularity, and $C_{2}$-cofiniteness, Trans. Amer. Math. Soc. 356 (2004), 3391-3402. MR2052955 (2005c:17041)

[BPZ] A. A. Belavin, A. M. Polyakov and A. B. Zamolodchikov, Infinite conformal symmetries in two-dimensional quantum field theory, Nucl. Phys. B241 (1984), 333-380. MR757857 $(86 \mathrm{~m}: 81097)$

[BH] A. E. Brouwer and W. H. Haemers, Spectra of Graphs, Universitext, Springer, 2012. MR2882891

[CIZ1] A. Cappelli, C. Itzykson and J.-B. Zuber, Modular invariant partition function in two dimensions, Nucl. Phys. B280 (1987), 445-464. MR881119 (88i:81132)

[CIZ2] A. Cappelli, C. Itzykson and J.-B. Zuber, The $A-D-E$ classification of minimal and $A_{1}^{(1)}$ conformal invariant theories, Comm. Math. Phys. 113 (1987), 1-26. MR918402 (89b:81178)

[C] R. Coquereaux, Global dimensions for Lie groups at level $k$ and their conformally exceptional quantum subgroups, Revista de la Union Matematica Argentina 51 (2010), No 2, 17-42. MR2840162

[DVVV] R. Dijkgraaf, C. Vafa, E. Verlinde and H. Verlinde, The operator algebra of orbifold models, Comm. Math. Phys. 123 (1989), 485-526. MR1003430 (91c:81132)

[D] C. Dong, Vertex algebras associated with even lattices, J. Alg. 161 (1993), 245-265. MR1245855 (94j:17023)

[DG] C. Dong and R. Jr. Griess, Rank one lattice type vertex operator algebras and their automorphism groups, J. Alg. 208 (1998), 262-275. MR1644007 (99h:17029)

[DGH] C. Dong, R. Jr. Griess and G. Hoehn, Framed vertex operator algebras, codes and the Moonshine module, Comm. Math. Phys. 193 (1998), 407-448. MR1618135 (99g:17050)

[DJ] C. Dong and C. Jiang, A characterization of vertex operator algebra $L\left(\frac{1}{2}, 0\right) \otimes L\left(\frac{1}{2}, 0\right)$, Comm. Math. Phys. 296 (2010), 69-88. MR2606628(2011c:17049)

[DL] C. Dong and J. Lepowsky, Generalized Vertex Algebras and Relative Vertex Operators, Progress in Math, Vol. 112, Birkhäuser, Boston, 1993. MR1233387(95b:17032)

[DLM1] C. Dong, H. Li and G. Mason, Compact automorphism groups of vertex operator algebras, Int. Math. Res. Not. 18 (1996), 913-921. MR.1420556 (98a:17044)

[DLM2] C. Dong, H. Li and G. Mason, Regularity of rational vertex operator algebras, Adv. Math. 132 (1997), 148-166. MR:1488241 (98m:17037)

[DLM3] C. Dong, H. Li and G. Mason, Twisted representations of vertex operator algebras, Math. Ann. 310 (1998), 571-600. MR.1615132(99d:17030)

[DLM4] C. Dong, H. Li and G. Mason, Vertex operator algebras and associative algebras, J. Alg. 206 (1998), 67-96. MR1637252 (99i:17029)

[DLM5] C. Dong, H. Li and G. Mason, Modular-invariance of trace functions in orbifold theory and generalized moonshine, Comm. Math. Phys. 214 (2000), 1-56. MR.1794264 (2001k:17043)

[DM1] C. Dong and G. Mason, On quantum Galois theory, Duke Math. J. 86 (1997), 305-321. MR:1430435 (97k:17042)

[DM2] C. Dong and G. Mason, Quantum Galois theory for compact Lie groups, J. Alg. 214 (1999), 92-102. MR,1684904 (2000g:17043b)

[DM3] C. Dong and G. Mason, Rational vertex operator algebras and the effective central charge, Int. Math. Res. Not. 56 (2004), 2989-3008. MR2097833 (2005k:17034)

[DMZ] C. Dong, G. Mason and Y. Zhu, Discrete series of the Virasoro algebra and the moonshine module, Proc. Symp. Pure. Math, Amer. Math. Soc. 56 II, (1994), 295-316. MR.1278737 (95c:17043)

[DY] C. Dong and N. Yu, Z-graded weak modules and regularity, Comm. Math. Phys. 316 (2012), 269-277. MR2989460

[ENO] P. Etingof, D. Nikshych and V. Ostrik, On fusion categories, Ann. Math. (2) 162 (2005), 581-642. MR:2183279 (2006m:16051)

[FMS] P. D. Francesco, P. Mathieu and D. Snchal, Conformal field theory, Springer-Verlag, 1997. MR 1424041 (97g:81062)

[FHL] I. Frenkel, Y. Huang and J. Lepowsky, On axiomatic approaches to vertex operator algebras and modules, Mem. Amer. Math. Soc. 104 1993. MR.1142494 (94a:17007) 
[FLM] I. Frenkel, J. Lepowsky and A. Meurman, Vertex Operator Algebras and the Monster, Pure and Applied Math., Vol. 134, Academic Press, Boston, 1988. MR996026 (90h:17026)

[FZ] I. Frenkel and Y. Zhu, Vertex operator algebras associated to representations of affine and Virasoro algebras, Duke Math. J. 66 (1992), 123-168. MR.1159433 (93g:17045)

[GL] D. Guido, and R. Longo, An algebraic spin and statistics theorem, Comm. Math. Phys. 172 (1995), 517-533. MR 1354258 (97i:81068)

[HMT] A. Hanaki, M. Miyamoto, and D. Tambara, Quantum Galois theory for finite groups, Duke Math. J. 97 (1999), 541-544. MR1682988(2000g:17043a)

[H] Y.-Z. Huang, Vertex operator algebras and the Verlinde Conjecture, Comm. Contemp. Math. 10 (2008), 103-154. MR2387861(2009e:17056)

[HL1] Y.-Z. Huang and J. Lepowsky, Toward a theory of tensor product for representations of a vertex operator algebra, in Proc. 20th Intl. Conference on Diff. Geom. Methods in Theoretical Physics, New York, 1991, ed. S. Catto and A. Rocha, World Scientific, Singapore, 1992, Vol. 1, 344-354. MR.1225125 (94k:17045)

[HL2] Y.-Z. Huang and J. Lepowsky, Tensor products of modules for a vertex operator algebra and vertex tensor categories, Lie Theory and Geometry, Birkhauser, Boston, 1994, 349383. MR $1327541(96 \mathrm{e}: 17061)$

[HL3] Y.-Z. Huang and J. Lepowsky, A theory of tensor products for module categories for a vertex operator algebra, I, Sel. Math. 1 (1995), 699-756. MR1383584(98a:17047)

[HL4] Y.-Z. Huang and J. Lepowsky, A theory of tensor products for module categories for a vertex operator algebra, III, J. Pure Appl. Alg. 100 (1995), 141-171. MR.1344848 (98a:17049)

[IZ] C. Itzykson and J.-B. Zuber, Two-dimensional conformal invariant theories on a torus, Nucl. Phys. B27, (1986), 580-616. MR865230 (88f:81111)

[J] V.F.R. Jones, Index for subfactors, Inv. Math. 72 (1983), 1-25. MR696688(84d:46097)

[K] V. G. Kac, Infinite-dimensional Lie algebras, Third edition, Cambridge University Press, Cambridge, 1990. MR 1104219 (92k:17038)

[KR] V. G. Kac and A. Raina, Highest Weight Representations of Infinite Dimensional Lie Algebras, World Scientific, Adv. Ser. In Math. Phys. Singapore, 1987. MR1021978 (90k:17013)

[Ka] M. Kaku, Strings, Conformal Fields, and Topology, Grad. Texts in Contemp. Phys. Springer-Verlag, New York, 1991. MR.1102894 (92i:81288)

[KLM] Y. Kawahigashi, R. Longo and M. Müger, Multi-interval subfactors and modularity of representations in conformal field theory, Comm. Math. Phys. 219 (2001), 631-669. MR.1838752 (2002g:81059)

[LS] P. W. H. Lemmens and J. J. Seidel, Equiangular lines, J. Alg. 24 (1973), 494-512. MR0307969 (46:7084)

[Li1] H. Li, Local systems of vertex operators, vertex superalgebras and modules, J. Pure Appl. Alg. 109 (1996), 143-195. MR.1387738 (97d:17016)

[Li2] H. Li, An analogue of the Hom functor and a generalized nuclear democracy theorem, Duke Math. J. 93 (1998), 73-114. MR1620083 (99d:17031)

[M] A. Milas, Fusion rings for degenerate minimal models, J. Alg. 254 (2002), 300-335. MR1933872(2003k:17037)

[MS] G. Moore and N. Seiberg, Classical and quantum conformal field theory, Comm. Math. Phys. 123 (1989), 177-254. MR.1002038(90e:81216)

[PP] M. Pimsner and S. Popa, Entropy and index for subfactors, Ann. Sci. Ec. Norm. Sup. 19 (1986), 57-106. MR860811 (87m:46120)

[S] J. H. Smith, Some properties of the spectrum of a graph, Combinatorial Structures and their Applications (Proc. Calgary Internat. Conf., Calgary, Alta.), pp. 403-406, New York: Gordon and Breach, 1970. MR0266799 (42:1702)

[V] E. Verlinde, Fusion rules and modular transformation in 2D conformal field theory, Nucl. Phys. B300 (1988), 360-376. MR954762(89h:81238)

[W] W. Wang, Rationality of Virasoro vertex operator algebras, Duke Math. J. 71, (1993), 197-211. MR 1230296 (94i:17034)

[Wa] A. Wassermann, Operator algebras and conformal field theory III: Fusion of positive energy representations of $L S U(N)$ using bounded operators, Inv. Math. 133 (1998), 467-538. MR 1645078(99j:81101) 
[X] F. Xu, Algebraic orbifold conformal field theories, Proc. Natl. Acad. Sci. USA 97 (2000), 14069-14073. MR:1806798(2002a:81156)

[Xu $\mathrm{X}$. Xu, Introduction to Vertex Operator Superalgebras and Their Modules, Mathematics and its Applications, Vol. 456, Kluwer Academic Publishers, Dordrecht, 1998. MR:1656671 (2000h:17019)

[Z] Y. Zhu, Modular invariance of characters of vertex operator algebras, J. Amer, Math. Soc. 9 (1996), 237-302. MR1317233 (96c:17042)

Department of Mathematics, University of California, Santa Cruz, California 95064

Department of Mathematics, University of California, Santa Cruz, California 95064

Department of Mathematics, University of California, Riverside, California 92521 